\title{
EQUI-INTEGRABILITY RESULTS FOR 3D-2D DIMENSION REDUCTION PROBLEMS
}

\author{
Marian BoceA $^{1}$ And IREne FonsecA ${ }^{1}$
}

\begin{abstract}
D-2D asymptotic analysis for thin structures rests on the mastery of scaled gradients $\left(\nabla_{\alpha} u_{\varepsilon} \mid \frac{1}{\varepsilon} \nabla_{3} u_{\varepsilon}\right)$ bounded in $L^{p}\left(\Omega ; \mathbb{R}^{9}\right), 1<p<+\infty$. Here it is shown that, up to a subsequence, $u_{\varepsilon}$ may be decomposed as $w_{\varepsilon}+z_{\varepsilon}$, where $z_{\varepsilon}$ carries all the concentration effects, i.e. $\left\{\left|\left(\nabla_{\alpha} w_{\varepsilon} \mid \frac{1}{\varepsilon} \nabla_{3} w_{\varepsilon}\right)\right|^{p}\right\}$ is equi-integrable, and $w_{\varepsilon}$ captures the oscillatory behavior, i.e. $z_{\varepsilon} \rightarrow 0$ in measure. In addition, if $\left\{u_{\varepsilon}\right\}$ is a recovering sequence then $z_{\varepsilon}=z_{\varepsilon}\left(x_{\alpha}\right)$ nearby $\partial \Omega$.
\end{abstract}

Mathematics Subject Classification. 49J45, 74B20, 74G10, 74K15, 74K35.

Received February 7, 2002.

\section{INTRODUCTION}

The treatment of nonlinear 3D-2D dimensional reduction has been undertaken successfully in recent years via asymptotic analysis in the Calculus of Variations (e.g. see $[3,7-9,11,12,15,18,24,25,32])$. Consider a homogeneous thin 3D domain with reference configuration $\Omega_{\varepsilon}:=\omega \times(-\varepsilon, \varepsilon)$, where $\omega$ is a bounded domain in $\mathbb{R}^{2}$, and whose elastic energy density is a continuous function $W: \mathbb{M}^{3 \times 3} \rightarrow \mathbb{R}$ satisfying the $p$ - growth and coercivity condition

$$
\frac{1}{C}\|A\|^{p}-C \leq W(A) \leq C\left(1+\|A\|^{p}\right)
$$

for some $1<p<+\infty$, where $C>0$ is a real constant and $\mathbb{M}^{3 \times 3}$ denotes the space of real $3 \times 3$ matrices endowed with the usual Euclidean norm $\|A\|:=\sqrt{\operatorname{tr}\left(A^{T} A\right)}$. The total energy of the film under a deformation $u: \Omega_{\varepsilon} \rightarrow \mathbb{R}^{3}$ is given by

$$
\tilde{E}_{\varepsilon}(u):=\int_{\Omega_{\varepsilon}} W(\nabla u(y)) \mathrm{d} y-\int_{\Omega_{\varepsilon}} f^{\varepsilon}(y) \cdot u(y) \mathrm{d} y,
$$

where $f^{\varepsilon} \in L^{p^{\prime}}\left(\Omega_{\varepsilon}, \mathbb{R}^{3}\right)$ stands for an appropriate dead loading body force density, and $p^{\prime}$ is the conjugate exponent of $p$, i.e. $\frac{1}{p}+\frac{1}{p^{\prime}}=1$.

We assume that the (quasi-static) equilibrium states of the film correspond to minimizers of $\tilde{E}_{\varepsilon}$ over the space of admissible deformations. To study the effective behavior of a very thin film, we consider a sequence $\left\{\varepsilon_{n}\right\}$ of

Keywords and phrases: Equi-integrability, dimension reduction, lower semicontinuity, maximal function, oscillations, concentrations, quasiconvexity.

1 Department of Mathematical Sciences, Carnegie Mellon University, Pittsburgh, PA 15213, USA;

e-mail: mbocea@andrew.cmu.edu 
positive real numbers (thickness) converging to zero and we rescale the problem by an $1 / \varepsilon_{n}$-dilatation in the transverse direction $x_{3}$ in order to recast energy functionals over varying domains $\Omega_{\varepsilon_{n}}$ into functionals with a fixed domain of integration. Precisely, performing the change of variables

$$
x_{1}=y_{1}, x_{2}=y_{2}, x_{3}=\frac{1}{\varepsilon_{n}} y_{3}
$$

and with $v(x):=u(y(x))$, we obtain

$$
\frac{1}{\varepsilon_{n}} \tilde{E}_{\varepsilon_{n}}(u)=E_{n}(v)
$$

where

$$
E_{n}(v):=\int_{\Omega} W\left(\nabla_{\alpha} v(x) \mid \frac{1}{\varepsilon_{n}} \nabla_{3} v(x)\right) \mathrm{d} x-\int_{\Omega} f^{\varepsilon_{n}}\left(x_{\alpha}, \varepsilon_{n} x_{3}\right) \cdot v(x) \mathrm{d} x
$$

$\Omega:=\omega \times(-1,1), \nabla_{\alpha} v$ is the $3 \times 2$ matrix of partial derivatives $\frac{\partial u_{i}}{\partial x_{\alpha}}, i \in\{1,2,3\}, \alpha \in\{1,2\}$, and $(A \mid a)$ denotes a $3 \times 3$ matrix whose first two columns are those of the $3 \times 2$ matrix $A$ and the last column is the vector $a \in \mathbb{R}^{3}$.

As explained in [18], the simplest assumption on the rescaled body force density $f^{\varepsilon_{n}}\left(x_{\alpha}, \varepsilon_{n} x_{3}\right)$ so as to be of order $O(1)$ and, together with the total bulk energy, to entail a nonlinear membrane behavior in the zero thickness limit is that $f^{\varepsilon_{n}}\left(x_{\alpha}, \varepsilon_{n} x_{3}\right)$ be independent of $n$. Thus, the study of the effective energy of the limiting system rests on the limit of the energies $I_{\varepsilon_{n}}\left(v_{n}\right):=\int_{\Omega} W\left(\nabla_{\alpha} v_{n} \mid \frac{1}{\varepsilon_{n}} \nabla_{3} v_{n}\right) \mathrm{d} x$, where $v_{n}$ is a minimizer of $E_{n}$. In view of (1.1), for energy bounded sequences we have the a priori bound

$$
\sup _{n \in \mathbb{N}} \int_{\Omega}\left|\left(\nabla_{\alpha} v_{n} \mid \frac{1}{\varepsilon_{n}} \nabla_{3} v_{n}\right)\right|^{p}\left(x_{\alpha}, x_{3}\right) \mathrm{d} x_{\alpha} \mathrm{d} x_{3}<+\infty
$$

and, up to a subsequence, Poincaré-Friedrichs inequality entails the weak convergence in $W^{1, p}\left(\Omega ; \mathbb{R}^{3}\right)$ of the sequence $\left\{v_{n}-\int_{\Omega} v_{n}(y) \mathrm{d} y\right\}$ to a deformation $v \in W^{1, p}\left(\Omega ; \mathbb{R}^{3}\right)$, where $v=v\left(x_{\alpha}\right)$. Due to the possible presence of oscillations in the sequence of scaled gradients $\left\{\left(\nabla_{\alpha} v_{n} \mid \frac{1}{\varepsilon_{n}} \nabla_{3} v_{n}\right)\right\}$, it may happen that

$$
\lim _{n \rightarrow \infty} \int_{\Omega} W\left(\nabla_{\alpha} v_{n} \mid \frac{1}{\varepsilon_{n}} \nabla_{3} v_{n}\right) \mathrm{d} x \neq \int_{\Omega} W\left(\nabla_{\alpha} v \mid 0\right) \mathrm{d} x
$$

The characterization of (oscillatory) limits of nonlinear quantities in the Calculus of Variations has been analyzed in several contexts by means of Young measures. Young measures were introduced in Optimal Control Theory by Young in connection to nonconvex problems, thus providing the appropriate framework for the description of generalized minimizers in the Calculus of Variations (see [33,34]). Later Tartar developed the use of Young measures in the PDE framework (see [29-31]). For a detailed study of Young measures, we refer the reader to $[4-6,17,20-23,27]$, among others. It turns out that the ability to determine $\lim _{n \rightarrow+\infty} \int_{\Omega} W\left(\nabla_{\alpha} v_{n} \mid \frac{1}{\varepsilon_{n}} \nabla_{3} v_{n}\right) \mathrm{d} x$ in terms of parametrized probability measures is restricted to the case where it is apriori known that the sequence $\left\{W\left(\nabla_{\alpha} v_{n} \mid \frac{1}{\varepsilon_{n}} \nabla_{3} v_{n}\right)\right\}$ is equi-integrable, or, in light of (1.1), that $\left\{\left|\left(\nabla_{\alpha} v_{n} \mid \frac{1}{\varepsilon_{n}} \nabla_{3} v_{n}\right)\right|^{p}\right\}$ is equi-integrable. 
While this cannot be guaranteed in general for the original sequence $\left\{v_{n}\right\}$, we are able to show that, up to a subsequence (not relabelled), it is possible to decompose $\left\{\left(\nabla_{\alpha} v_{n} \mid \frac{1}{\varepsilon_{n}} \nabla_{3} v_{n}\right)\right\}$ as a sum of a sequence $\left\{\left(\nabla_{\alpha} w_{n} \mid \frac{1}{\varepsilon_{n}} \nabla_{3} w_{n}\right)\right\}$ whose $p$-th power is equi-integrable and a remainder that converges to zero in measure. We may say that $\left\{\left(\nabla_{\alpha} w_{n} \mid \frac{1}{\varepsilon_{n}} \nabla_{3} w_{n}\right)\right\}$ carries the oscillations, while the remainder accounts for the concentration effects. The main objective of this paper is to prove this decomposition result, controlling the uniform smallness of $\left\{\left(\nabla_{\alpha} w_{n} \mid \frac{1}{\varepsilon_{n}} \nabla_{3} w_{n}\right)\right\}$ on small sets by means of the initial sequence only. An alternative argument using De La Vallée-Poussin criterion has been recently communicated to the authors by Braides (see [10]).

Theorem 1.1. Let $\Omega:=\omega \times(-1,1)$, where $\omega \subset \mathbb{R}^{2}$ is an open, bounded Lipschitz domain, let $\left\{\varepsilon_{n}\right\}$ be a sequence of positive real numbers converging to zero, and let $\left\{v_{n}\right\}$ be a bounded sequence in $W^{1, p}\left(\Omega, \mathbb{R}^{3}\right)(1<p<+\infty)$ satisfying

$$
\sup _{n \in \mathbb{N}} \int_{\Omega}\left|\left(\nabla_{\alpha} v_{n} \mid \frac{1}{\varepsilon_{n}} \nabla_{3} v_{n}\right)\right|^{p}\left(x_{\alpha}, x_{3}\right) \mathrm{d} x_{\alpha} \mathrm{d} x_{3}<+\infty .
$$

Suppose further that $v_{n} \rightarrow v$ in $W^{1, p}\left(\Omega, \mathbb{R}^{3}\right)$ and $\frac{1}{\varepsilon_{n}} \nabla_{3} v_{n} \rightarrow b$ in $L^{p}\left(\Omega, \mathbb{R}^{3}\right)$. Then there exists a subsequence $\left\{v_{n_{k}}\right\}$ of $\left\{v_{n}\right\}$ and a sequence $\left\{w_{k}\right\} \subset W^{1, \infty}\left(\Omega, \mathbb{R}^{3}\right)$ such that

(i) $\lim _{k \rightarrow \infty} \mathcal{L}^{3}\left(\left\{x \in \Omega: w_{k}(x) \neq v_{n_{k}}(x)\right\}\right)=0$;

(ii) $\left\{\left(\nabla_{\alpha} w_{k} \mid \frac{1}{\varepsilon_{n_{k}}} \nabla_{3} w_{k}\right)\right\}$ is p-equi-integrable;

(iii) $w_{k} \rightarrow v$ weakly in $W^{1, p}\left(\Omega, \mathbb{R}^{3}\right)$;

(iv) $\frac{1}{\varepsilon_{n_{k}}} \nabla_{3} w_{k} \rightarrow b$ weakly in $L^{p}\left(\Omega, \mathbb{R}^{3}\right)$.

The corresponding decomposition result for the case of unscaled gradients has received much attention in recent years, due to the central role that it plays in a number of key results in the Calculus of Variations, among them the celebrated lower semicontinuity result of Acerbi and Fusco [1], the characterization of $W^{1, p}$ Young measures by Kinderlehrer and Pedregal [20,21], as well as numerous relaxation results for nonconvex integrands. While the technique needed to establish the decomposition property was essentially used in [1], the result was first isolated only ten years later in [22]. The proof in [22] is based on estimates for perturbed Hodge decomposition [19], and more recently Fonseca et al. [17] proposed another proof using $L^{p}$ estimates for maximal functions, Lipschitz extensions of $W^{1, p}$ functions off small sets, and Young measures. We follow a similar argument, although the degeneracy of the coercivity condition in the $x_{3}$ - direction leads us to repeat $v_{n}$ periodically on an infinite strip of copies of $\Omega$ in the $x_{3}$ - direction, thus obtaining non-degenerate uniform bounds for the resulting vertically periodized sequence. De Giorgi's slicing method will now come into play to help us selecting, via an averaging process, one of these layers with small energy concentration. Up to a subsequence, the restriction of $v_{n}$ to this copy of $\Omega$ will eventually become the new $w_{n}$.

As a first consequence of Theorem 1.1, we show that the Dirichlet problem on an arbitrarily large cylinder for fixed affine lateral boundary conditions

$$
\min \left\{\int_{\omega \times(-L, L)} W(\nabla v) \mathrm{d} x: v \in W^{1, p}\left(\omega \times(-L, L) ; \mathbb{R}^{3}\right), v=v_{0} \text { on } \partial \omega \times(-L, L)\right\},
$$

admits $p$-equi-integrable minimizing sequences energetically prefering thinner and thinner reference domains. Precisely, after changing variables as in (1.2), 
Corollary 1.2. Let $\Omega:=\omega \times(-1,1)$, where $\omega \subset \mathbb{R}^{2}$ is an open, bounded Lipschitz domain. Let $u_{0} \in W^{1, p}\left(\omega ; \mathbb{R}^{3}\right)$ be an affine mapping $(1<p<+\infty)$, and let $W: \mathbb{M}^{3 \times 3} \rightarrow \mathbb{R}$ be a continuous function satisfying (1.1). Define

$$
Q_{\omega}^{*} W\left(u_{0}\right):=\inf _{(u, L)}\left\{\frac{1}{\mathcal{L}^{3}(\Omega)} \int_{\Omega} W\left(\nabla_{\alpha} u \mid L \nabla_{3} u\right) \mathrm{d} x: L>0, u \in W^{1, p}\left(\Omega ; \mathbb{R}^{3}\right), u_{\mid \partial \omega \times(-1,1)}=u_{0}\right\} .
$$

Then

$$
Q_{\omega}^{*} W\left(u_{0}\right)=Q \bar{W}\left(\xi_{0}\right),
$$

where $\bar{W}(\xi):=\min _{z \in \mathbb{R}^{3}} W(\xi \mid z)$, for all $\xi \in \mathbb{M}^{3 \times 2}$, and $u_{0}\left(x_{\alpha}\right)=\xi_{0} x_{\alpha}$ for some $\xi_{0} \in \mathbb{M}^{3 \times 2}$. Moreover, given any sequence $\left\{\varepsilon_{n}\right\}$ of positive real numbers converging to zero, there exist a subsequence (not relabelled) of $\left\{\varepsilon_{n}\right\}$, and a sequence $\left\{u_{n}\right\} \subset W^{1, p}\left(\Omega ; \mathbb{R}^{3}\right)$ such that

(i) $\lim _{n \rightarrow \infty} \frac{1}{\mathcal{L}^{3}(\Omega)} \int_{\Omega} W\left(\nabla_{\alpha} u_{n} \mid \frac{1}{\varepsilon_{n}} \nabla_{3} u_{n}\right) \mathrm{d} x=Q \bar{W}\left(\xi_{0}\right)$;

(ii) $\lim _{n \rightarrow \infty}\left\|u_{n}-u_{0}\right\|_{L^{p}\left(\Omega ; \mathbb{R}^{3}\right)}=0$;

(iii) $u_{n_{\mid \partial \omega \times(-1,1)}}=u_{0}$;

(iv) $\left\{\left(\nabla_{\alpha} u_{n} \mid \frac{1}{\varepsilon_{n}} \nabla_{3} u_{n}\right)\right\}$ is p-equi-integrable.

Note that in view of (2.5) below, the inequality

$$
Q_{\omega}^{*} W\left(u_{0}\right) \leq \frac{1}{\mathcal{L}^{2}(\omega)} \int_{\omega} Q \bar{W}\left(\nabla_{\alpha} u_{0}\left(x_{\alpha}\right)\right) \mathrm{d} x_{\alpha},
$$

is valid for any $u_{0} \in W^{1, p}\left(\omega ; \mathbb{R}^{3}\right)$ not necessarily affine. However, in general the opposite inequality may fail. Indeed, as an example consider $W(\xi):=|\xi|^{2}$, let $\varphi_{0}$ be any function in $W^{1,2}(\omega)$ and set $u_{0}\left(x_{\alpha}\right):=\left(\varphi_{0}\left(x_{\alpha}\right), 0,0\right)$. Here $Q \bar{W}\left(\nabla_{\alpha} u_{0}\right)=\left|\nabla_{\alpha} \varphi_{0}\right|^{2}$, and if

$$
Q_{\omega}^{*} W\left(u_{0}\right)=\frac{1}{\mathcal{L}^{2}(\omega)} \int_{\omega} Q \bar{W}\left(\nabla_{\alpha} u_{0}\left(x_{\alpha}\right)\right) \mathrm{d} x_{\alpha}
$$

was satisfied then $\varphi_{0}$ would need to be harmonic in $\omega$.

Finally, in the last part of the paper we prove that energy recovering sequences $\left\{\left(v_{n}, \varepsilon_{n}\right)\right\}$ (see Def. 2.3) may be modified on a small set (up to a subsequence) so as to obtain a new recovering sequence which depends only on $x_{\alpha}$ nearby $\partial \Omega$. We view this result as a first step towards matching recovering sequences to their limits $u_{0}$ nearby $\partial \Omega$. This strategy (two-step matching) was previously introduced by Conti et al. in the context of second-order phase transitions (see [13]). The ability to match recovering sequences $\left\{\left(v_{n}, \varepsilon_{n}\right)\right\}$ to their limiting configurations $u_{0}$, would enable us to periodize $\left(v_{n}-u_{0}\right)$ on a cylindrical cell and, as a consequence, the additional symmetry hypothesis $W\left(F_{\alpha} \mid F_{3}\right)=W\left(F_{\alpha} \mid-F_{3}\right)$ considered in, say [8], would no longer be needed.

Corollary 1.3. Let $\Omega:=\omega \times(-1,1)$, where $\omega \subset \mathbb{R}^{2}$ is an open, bounded Lipschitz domain. Let $u_{0} \in$ $W^{1, p}\left(\omega ; \mathbb{R}^{3}\right)(1<p<+\infty)$ be given and let $W: \mathbb{M}^{3 \times 3} \rightarrow \mathbb{R}$ be a continuous function satisfying (1.1). Consider $\left\{\left(v_{n}, \varepsilon_{n}\right)\right\}$ to be a recovering sequence in the sense of Definition 2.3. Then there exists a subsequence $\left\{v_{n_{k}}\right\}$ of $\left\{v_{n}\right\}$ and a sequence $\left\{w_{k}\right\} \subset W^{1, p}\left(\Omega ; \mathbb{R}^{3}\right)$ such that

(i) $\lim _{n \rightarrow \infty} \mathcal{L}^{3}\left(\left\{x \in \Omega: w_{k}(x) \neq v_{n_{k}}(x)\right\}\right)=0$;

(ii) $\left\{\left(\nabla_{\alpha} w_{k} \mid \frac{1}{\varepsilon_{n_{k}}} \nabla_{3} w_{k}\right)\right\}$ is p-equi-integrable; 
(iii) $w_{k}=w_{k}\left(x_{\alpha}\right)$ nearby $x_{3}= \pm 1$;

(iv) $\left\{\left(w_{k}, \varepsilon_{n_{k}}\right)\right\}$ is still a recovering sequence.

\section{Preliminaries}

We first recall some well-known facts about maximal functions (see [28] for details). Given a Borel measurable function $u: \mathbb{R}^{N} \rightarrow \mathbb{R}^{d}$, the maximal function of $u$ is defined by

$$
M(u)(x):=\sup _{r>0} \frac{1}{\mathcal{L}^{N}(B(x, R))} \int_{B(x, R)}|u(y)| \mathrm{d} y .
$$

If $u \in L^{p}\left(\mathbb{R}^{N} ; \mathbb{R}^{m}\right)$ with $p>1$, then $|u(x)| \leq M(u)(x)$ for $\mathcal{L}^{N}$ a.e. $x \in \mathbb{R}^{N}$, and

$$
\|M(u)\|_{L^{p}\left(\mathbb{R}^{N}\right)} \leq C(N, p)\|u\|_{L^{p}\left(\mathbb{R}^{N} ; \mathbb{R}^{m}\right)} .
$$

We will make use of the following decomposition result:

Proposition 2.1. ( [17] Lemma 1.2) Let $\Omega \in \mathbb{R}^{N}$ be an open and bounded set and let $\left\{w_{n}\right\}$ be a bounded sequence in $W^{1, p}\left(\Omega ; \mathbb{R}^{m}\right)$. There exists a subsequence $\left\{w_{n_{k}}\right\}$ of $\left\{w_{n}\right\}$, and a sequence $\left\{z_{k}\right\} \subset W^{1, p}\left(\Omega ; \mathbb{R}^{m}\right)$ such that

$$
\lim _{k \rightarrow+\infty} \mathcal{L}^{N}\left(\left\{x \in \Omega: z_{k}(x) \neq w_{n_{k}}(x)\right\}\right)=0,
$$

and $\left\{\left|\nabla z_{k}\right|^{p}\right\}$ is equi-integrable. If $\Omega$ is Lipschitz (or, more generally, an extension domain), then each $z_{j}$ may be chosen to be a Lipschitz function.

The following Poincaré-type inequality is well known (see, e.g. [35]). We include a proof for the convenience of the reader.

Lemma 2.2. Let $\Omega \subset \mathbb{R}^{N}$ be an open, bounded, Lipschitz domain. Then for every $\alpha>0$ there exists $C(\alpha)>0$ such that

$$
\int_{\Omega}|u(x)|^{p} \mathrm{~d} x \leq C(\alpha) \int_{\Omega}|\nabla u(x)|^{p} \mathrm{~d} x
$$

for all $u \in W^{1, p}\left(\Omega, \mathbb{R}^{d}\right)$ satisfying

$$
\mathcal{L}^{N}(\{x \in \Omega: u(x)=0\}) \geq \alpha .
$$

Proof. Without loss of generality, we may assume that $\mathcal{L}^{N}(\Omega)=1$. If the result was false, then there would exist $\alpha>0$ and a sequence $\left\{u_{n}\right\} \subset W^{1, p}\left(\Omega, \mathbb{R}^{d}\right)$ such that $\mathcal{L}^{N}\left(\left\{x \in \Omega: u_{n}(x)=0\right\}\right) \geq \alpha$ and

$$
\int_{\Omega}\left|u_{n}(x)\right|^{p} \mathrm{~d} x>n \int_{\Omega}\left|\nabla u_{n}(x)\right|^{p} \mathrm{~d} x .
$$

Define

$$
w_{n}:=\frac{u_{n}}{\left\|u_{n}\right\|_{L^{p}\left(\Omega, \mathbb{R}^{d}\right)}}
$$


and note that $\left\|w_{n}\right\|_{L^{p}\left(\Omega, \mathbb{R}^{d}\right)}=1, \mathcal{L}^{N}\left(\left\{x \in \Omega: w_{n}(x)=0\right\}\right) \geq \alpha$. By (2.2) we obtain that

$$
\int_{\Omega}\left|\nabla w_{n}(x)\right|^{p} \mathrm{~d} x \rightarrow 0 \text { as } n \rightarrow \infty
$$

and thus the sequence $\left\{w_{n}\right\}$ is bounded in $W^{1, p}\left(\Omega, \mathbb{R}^{d}\right)$. Up to a subsequence (not relabelled) we have $w_{n} \rightarrow w$ weakly in $W^{1, p}\left(\Omega, \mathbb{R}^{d}\right)$ and $w_{n} \rightarrow w$ strongly in $L^{p}\left(\Omega, \mathbb{R}^{d}\right)$, for some $w \in W^{1, p}\left(\Omega, \mathbb{R}^{d}\right)$. We deduce that $\nabla w=0 \mathcal{L}^{N}$ a.e. in $\Omega$, and since $\|w\|_{L^{p}\left(\Omega, \mathbb{R}^{d}\right)}=1$ we have that $w=c \mathcal{L}^{N}$ a.e. in $\Omega$, where $|c|=1$. Since $w_{n} \rightarrow w$ in measure, we have

$$
\lim _{n \rightarrow \infty} \mathcal{L}^{N}\left(\left\{x \in \Omega:\left|w_{n}(x)-c\right|>\frac{1}{2}\right\}\right)=0
$$

and thus

$$
0<\alpha \leq \liminf _{n \rightarrow \infty} \mathcal{L}^{N}\left(\left\{x \in \Omega: w_{n}(x)=0\right\}\right) \leq \lim _{n \rightarrow \infty} \mathcal{L}^{N}\left(\left\{x \in \Omega:\left|w_{n}(x)-c\right|>\frac{1}{2}\right\}\right)=0 .
$$

We have reached a contradiction.

Let $W: \mathbb{M}^{3 \times 3} \rightarrow \mathbb{R}$ be a continuous function satisfying (1.1). We define the two-dimensional energy density $\bar{W}: \mathbb{M}^{3 \times 2} \rightarrow \mathbb{R}$, by

$$
\bar{W}(\xi):=\min _{z \in \mathbb{R}^{3}} W(\xi \mid z), \quad \xi \in \mathbb{M}^{3 \times 2} .
$$

The function $\bar{W}$ is continuous and satisfies growth and coercivity estimates similar to (1.1) (see [24]). We will denote by $Q \bar{W}$ the quasiconvex envelope of $\bar{W}$, precisely

$$
Q \bar{W}(\eta):=\inf \left\{\frac{1}{\mathcal{L}^{2}(\omega)} \int_{\omega} \bar{W}\left(\eta+\nabla_{\alpha} \varphi\left(x_{\alpha}\right)\right) \mathrm{d} x_{\alpha}: \varphi \in W_{0}^{1, \infty}\left(\omega ; \mathbb{R}^{3}\right)\right\}, \eta \in \mathbb{M}^{3 \times 2},
$$

where we have used the well known fact that quasiconvex envelope of $\bar{W}$ is independent of the domain of integration in (2.4) (see [14]).

Using $\Gamma$ - convergence techniques, it has been shown by Le Dret and Raoult [24] (see also $[8,9,11,12,15]$ ) that $Q \bar{W}$ is the effective two-dimensional asymptotic thin film energy for the family $\left\{I_{\varepsilon}\right\}$. Indeed, if $u_{0} \in$ $W^{1, p}\left(\omega ; \mathbb{R}^{3}\right), u_{0}=u_{0}\left(x_{\alpha}\right)$, then

$$
\begin{aligned}
& \frac{1}{\mathcal{L}^{2}(\omega)} \int_{\omega} Q \bar{W}\left(\nabla_{\alpha} u_{0}\left(x_{\alpha}\right)\right) \mathrm{d} x_{\alpha}=\inf \left\{\liminf _{n \rightarrow \infty} \frac{1}{\mathcal{L}^{3}(\Omega)} \int_{\Omega} W\left(\nabla_{\alpha} u_{n} \mid \frac{1}{\varepsilon_{n}} \nabla_{3} u_{n}\right) \mathrm{d} x:\right. \\
& \left.\varepsilon_{n} \rightarrow 0^{+}, u_{n} \in W^{1, p}\left(\Omega ; \mathbb{R}^{3}\right), \lim _{n \rightarrow \infty}\left\|u_{n}-u_{0}\right\|_{L^{p}\left(\Omega ; \mathbb{R}^{3}\right)}=0, u_{n \mid \partial \omega \times(-1,1)}=u_{0}\right\} .
\end{aligned}
$$

Definition 2.3. A sequence $\left\{\left(v_{n}, \varepsilon_{n}\right)\right\} \subset W^{1, p}\left(\Omega ; \mathbb{R}^{3}\right) \times(0,+\infty)$ is called a recovering sequence if $\varepsilon_{n} \rightarrow$ $0^{+}, v_{n \mid \partial \omega \times(-1,1)}=u_{0}, \lim _{n \rightarrow \infty}\left\|v_{n}-u_{0}\right\|_{L^{p}\left(\Omega ; \mathbb{R}^{3}\right)}=0$, and if

$$
\lim _{n \rightarrow \infty} \int_{\Omega} W\left(\nabla_{\alpha} v_{n} \mid \frac{1}{\varepsilon_{n}} \nabla_{3} v_{n}\right) \mathrm{d} x=2 \int_{\omega} Q \bar{W}\left(\nabla_{\alpha} u_{0}\left(x_{\alpha}\right)\right) \mathrm{d} x_{\alpha} .
$$


Remark 2.4. Using the definition of the infimum in (2.5) and a standard diagonalization argument, it can be shown that a recovering sequence in the sense of the Definition 2.3 always exists.

\section{Proof of Theorem 1.1}

In what follows, and without loss of generality, we assume that $\varepsilon_{n} \in(0,1 / 12)$. Also, $C>0$ denotes a generic constant which may vary from line to line and expression to expression within the same formula.

Step 1: For every $n \in \mathbb{N}$, set $S_{i}^{(n)}:=\omega \times\left((2 i-1) \varepsilon_{n},(2 i+1) \varepsilon_{n}\right)$, with $i \in[-L(n), L(n)] \cap \mathbb{Z}$, where the number $L(n) \in \mathbb{N}$ of layers is given by

$$
L(n):= \begin{cases}\frac{1}{2 \varepsilon_{n}}, & \text { if } \frac{1}{2 \varepsilon_{n}} \in \mathbb{N} \\ {\left[\frac{1}{2 \varepsilon_{n}}\right]+1,} & \text { otherwise }\end{cases}
$$

Note that

$$
\frac{1}{\varepsilon_{n}} \leq 2 L(n) \leq 2+\frac{1}{\varepsilon_{n}}
$$

Define $u_{n}: S_{0}^{(n)} \rightarrow \mathbb{R}^{3}$ as $u_{n}\left(x_{\alpha}, x_{3}\right):=v_{n}\left(x_{\alpha}, \frac{1}{\varepsilon_{n}} x_{3}\right)$. We have, changing variables,

$$
\frac{1}{\varepsilon_{n}} \int_{S_{0}^{(n)}}\left|\nabla u_{n}(x)\right|^{p} \mathrm{~d} x=\int_{\Omega}\left|\left(\nabla_{\alpha} v_{n} \mid \frac{1}{\varepsilon_{n}} \nabla_{3} v_{n}\right)(y)\right|^{p} \mathrm{~d} y,
$$

and thus,

$$
\sup _{n \in \mathbb{N}} \frac{1}{\varepsilon_{n}} \int_{S_{0}^{(n)}}\left|\nabla u_{n}(x)\right|^{p} \mathrm{~d} x<+\infty
$$

Define $F_{i}^{(n)}: S_{i}^{(n)} \rightarrow S_{0}^{(n)}$ by

$$
F_{i}^{(n)}\left(x_{\alpha}, x_{3}\right):=\left(x_{\alpha},(-1)^{i} x_{3}+2(-1)^{i+1} i \varepsilon_{n}\right),
$$

set $\Omega_{n}:=\omega \times\left(-(2 L(n)+1) \varepsilon_{n},(2 L(n)+1) \varepsilon_{n}\right)$, and let $F_{n}: \Omega_{n} \rightarrow S_{0}^{(n)}$ be given by

$$
F_{n}(x):=\sum_{i=-L(n)}^{L(n)} \chi_{S_{i}^{(n)}}(x) F_{i}^{(n)}(x) \text { if } x \in \bigcup_{i=-L(n)}^{L(n)} S_{i}^{(n)},
$$

where $F_{n}$ is extended continuously to $\Omega_{n}$. We consider now the vertical periodization of $u_{n}$, precisely $\bar{u}_{n}: \Omega_{n} \rightarrow$ $\mathbb{R}^{3}$ defined as

$$
\bar{u}_{n}(x):=u_{n}\left(F_{n}(x)\right) .
$$

Using the fact that for every $n \in \mathbb{N}$ and $i \in[-L(n), L(n)] \cap \mathbb{Z}$,

$$
\nabla F_{i}^{(n)}=\left(\begin{array}{ccc}
1 & 0 & 0 \\
0 & 1 & 0 \\
0 & 0 & (-1)^{i}
\end{array}\right) \text { in } S_{i}^{(n)}
$$


by (3.1) we can estimate

$$
\begin{aligned}
\int_{\Omega}\left|\nabla \bar{u}_{n}(x)\right|^{p} \mathrm{~d} x & =\sum_{i=-L(n)}^{L(n)} \int_{S_{i}^{(n)} \cap \Omega}\left|\nabla u_{n}\left(F_{i}^{(n)}(x)\right)\right|^{p} \mathrm{~d} x \\
& \leq \sum_{i=-L(n)}^{L(n)} \int_{S_{0}^{(n)}}\left|\nabla u_{n}(y)\right|^{p} \mathrm{~d} y \leq\left(3+\frac{1}{\varepsilon_{n}}\right) \int_{S_{0}^{(n)}}\left|\nabla u_{n}(y)\right|^{p} \mathrm{~d} y .
\end{aligned}
$$

By virtue of (3.2),

$$
\sup _{n \in \mathbb{N}} \int_{\Omega}\left|\nabla \bar{u}_{n}(x)\right|^{p} \mathrm{~d} x<+\infty .
$$

A similar computation gives

$$
\sup _{n \in \mathbb{N}} \int_{\Omega}\left|\bar{u}_{n}(x)\right|^{p} \mathrm{~d} x \leq \frac{5}{4} \sup _{n \in \mathbb{N}} \int_{\Omega}\left|v_{n}(x)\right|^{p} \mathrm{~d} x<+\infty,
$$

and thus,

$$
\sup _{n \in \mathbb{N}}\left\|\bar{u}_{n}\right\|_{W^{1, p}\left(\Omega, \mathbb{R}^{3}\right)}<+\infty .
$$

In view of Lemma 2.1 , there exists a subsequence $\left\{\bar{u}_{n_{k}}\right\}$ of $\left\{\bar{u}_{n}\right\}$, and a sequence $\left\{z_{k}\right\} \subset W^{1, \infty}\left(\Omega ; \mathbb{R}^{m}\right)$ such that the sequence $\left\{\left|\nabla z_{k}\right|^{p}\right\}$ is equi-integrable and

$$
\lim _{k \rightarrow+\infty} \mathcal{L}^{3}\left(E_{k}\right)=0
$$

where

$$
E_{k}:=\left\{x \in \Omega: z_{k}(x) \neq \bar{u}_{n_{k}}(x)\right\}
$$

Define $\Lambda:(0, t) \rightarrow(0,+\infty)$ by

$$
\Lambda(t):=\sup \left\{\int_{A}\left|\nabla z_{m}(x)\right|^{p} \mathrm{~d} x: m \in \mathbb{N}, A \in \mathcal{B}(\Omega), \mathcal{L}^{3}(A) \leq t\right\},
$$

where $\mathcal{B}(\Omega)$ denotes the class of Borel subsets of $\Omega$. We note that the equi-integrability property of $\left\{\left|\nabla z_{k}\right|^{p}\right\}$ ensures that

$$
\lim _{t \rightarrow 0^{+}} \Lambda(t)=0 .
$$

Step 2: Set $I:=\left[-L\left(n_{k}\right), L\left(n_{k}\right)\right] \cap \mathbb{Z}$ and $I_{\star}:=\left[-L\left(n_{k}\right)+2, L\left(n_{k}\right)-2\right] \cap \mathbb{Z}$. Note that if $i \in I_{\star}$ then $S_{i}^{\left(n_{k}\right)} \subset \Omega$. By (3.1) we have

$$
\frac{1}{\varepsilon_{n_{k}}}-3 \leq \operatorname{card} I_{\star} \leq \frac{1}{\varepsilon_{n_{k}}}-1
$$


If for an infinite number of indices $k$ (not relabelled), we have that $\mathcal{L}^{3}\left(E_{k}\right)=0$, then given any Borel subset $A$ of $\Omega$ and setting $A_{k}:=\left\{y \in \Omega:\left(y_{\alpha}, y_{3}\right)=\left(x_{\alpha}, \varepsilon_{n_{k}} x_{3}\right), x \in A\right\} \subset S_{0}^{\left(n_{k}\right)}$ we can estimate

$$
\begin{aligned}
\int_{A}\left|\left(\nabla_{\alpha} v_{n_{k}} \mid \frac{1}{\varepsilon_{n_{k}}} \nabla_{3} v_{n_{k}}\right)\right|^{p} \mathrm{~d} x & =\frac{1}{\varepsilon_{n_{k}}} \int_{A_{k}}\left|\nabla u_{n_{k}}\right|^{p} \mathrm{~d} x \leq 2 \sum_{i \in I_{\star}} \int_{\left(F_{i}^{\left(n_{k}\right)}\right)^{-1}\left(A_{k}\right)}\left|\nabla \bar{u}_{n_{k}}\right|^{p} \mathrm{~d} x \\
& \left.=2 \int_{i \in I_{\star}}\left|\nabla z_{k}\right|^{p} \mathrm{~d} x \leq 2 \Lambda\left(\mathcal{L}^{\left(n_{k}\right)}\right)^{-1}(A)\right),
\end{aligned}
$$

where we have used $(3.3,3.6)$, and the fact that $\mathcal{L}^{3}\left(\bigcup_{i \in I_{\star}}\left(F_{i}^{\left(n_{k}\right)}\right)^{-1}\left(A_{k}\right)\right) \leq \mathcal{L}^{3}(A)$. In this case we conclude that $w_{k}:=v_{n_{k}}$ satisfies $(\mathrm{i}, \mathrm{ii})$.

Consider now the case where $\mathcal{L}^{3}\left(E_{k}\right)>0$ except maybe for a finite number of indices $k$. In what follows we will assume, without loss of generality, that $\mathcal{L}^{3}\left(E_{k}\right)>0$ for all $k \in \mathbb{N}$. Define

$$
I_{k}:=\left\{i \in I_{\star}: \mathcal{L}^{3}\left(E_{k} \cap S_{i}^{\left(n_{k}\right)}\right) \leq 2 \varepsilon_{n_{k}} \mathcal{L}^{3}\left(E_{k}\right)\right\}
$$

We have

$$
\mathcal{L}^{3}\left(E_{k}\right) \geq \mathcal{L}^{3}\left(\bigcup_{i \in I_{\star}}\left(E_{k} \cap S_{i}^{\left(n_{k}\right)}\right)\right) \geq \sum_{i \in I_{\star} \backslash I_{k}} \mathcal{L}^{3}\left(E_{k} \cap S_{i}^{\left(n_{k}\right)}\right) \geq 2 \varepsilon_{n_{k}} \mathcal{L}^{3}\left(E_{k}\right)\left(2 L\left(n_{k}\right)-3-\operatorname{card} I_{k}\right),
$$

and since $\mathcal{L}^{3}\left(E_{k}\right)>0$, by $(3.1)$ we find

$$
\operatorname{card} I_{k} \geq \frac{1}{2 \varepsilon_{n_{k}}}-3
$$

Without loss of generality, we may assume that all indices $i \in I_{k}$ are even and we replace now the above lower bound for card $I_{k}$ by

$$
\operatorname{card} I_{k} \geq \frac{1}{2}\left(\frac{1}{2 \varepsilon_{n_{k}}}-3\right)
$$

For each $i \in I_{k}$ define $G^{(i, k)}: \Omega_{n_{k}} \rightarrow S_{i}^{\left(n_{k}\right)}$ by

$$
G^{(i, k)}(x):=\sum_{j \in I} \chi_{S_{j}^{\left(n_{k}\right)}}(x) G_{j}^{(i, k)}(x) \text { if } x \in \bigcup_{j \in I} S_{j}^{\left(n_{k}\right)}
$$

where $G_{j}^{(i, k)}: S_{j}^{\left(n_{k}\right)} \rightarrow S_{i}^{\left(n_{k}\right)}$ is given by

$$
G_{j}^{(i, k)}(x):=\left(\left(F_{i}^{\left(n_{k}\right)}\right)^{-1} \circ F_{j}^{\left(n_{k}\right)}\right)(x)
$$

and $G^{(i, k)}$ is extended to the whole $\Omega_{n_{k}}$ by continuity.

Also, for each $i \in I_{k}$ set

$$
X^{(i, k)}:=\left\{x \in \Omega: z_{k}(x) \neq z_{k}\left(G^{(i, k)}(x)\right)\right\}
$$


Claim 1: For each $k \in \mathbb{N}$, there exists an index $i_{0} \in I_{k}$ and a nonnegative real number $a_{k}$ such that

$$
\sum_{j \in I_{k}} \int_{G_{j}^{\left(i_{0}, k\right)}\left(X^{\left(i_{0}, k\right)} \cap S_{j}^{\left(n_{k}\right)}\right)}\left|\nabla z_{k}(x)\right|^{p} \mathrm{~d} x \leq a_{k}, \text { and } \lim _{k \rightarrow+\infty} a_{k}=0 .
$$

We postpone the proof of Claim 1 until after Step 3 is completed.

Step 3: Assuming that the Claim 1 holds, define $w_{k}: \Omega \rightarrow \mathbb{R}^{3}$ by

$$
w_{k}(x):=z_{k}\left(\left(F_{i_{0}}^{\left(n_{k}\right)}\right)^{-1}\left(x_{\alpha}, \varepsilon_{n_{k}} x_{3}\right)\right),
$$

for each $k \in \mathbb{N}$, where the index $i_{0} \in I_{k}$ has been selected according to (3.8). We will first show that this sequence satisfies (i) and (ii).

For every $x, y \in \Omega$ we have

$$
\begin{aligned}
\left|w_{k}(x)-w_{k}(y)\right| & =\left|z_{k}\left(\left(F_{i_{0}}^{\left(n_{k}\right)}\right)^{-1}\left(x_{\alpha}, \varepsilon_{n_{k}} x_{3}\right)\right)-z_{k}\left(\left(F_{i_{0}}^{\left(n_{k}\right)}\right)^{-1}\left(y_{\alpha}, \varepsilon_{n_{k}} y_{3}\right)\right)\right| \\
& \leq \operatorname{Lip}\left(z_{k}\right)\left|\left(F_{i_{0}}^{\left(n_{k}\right)}\right)^{-1}\left(x_{\alpha}, \varepsilon_{n_{k}} x_{3}\right)-\left(F_{i_{0}}^{\left(n_{k}\right)}\right)^{-1}\left(y_{\alpha}, \varepsilon_{n_{k}} y_{3}\right)\right| \\
& \leq \operatorname{Lip}\left(z_{k}\right)|x-y|
\end{aligned}
$$

and thus $w_{k}$ is still a Lipschitz function and we have

$$
\operatorname{Lip}\left(w_{k}\right) \leq \operatorname{Lip}\left(z_{k}\right)
$$

To prove (i), define

$$
M_{k}:=\left\{x \in \Omega: w_{k}(x) \neq v_{n_{k}}(x)\right\}
$$

We have

$$
\begin{aligned}
\mathcal{L}^{3}\left(M_{k}\right) & =\mathcal{L}^{3}\left(\left\{x \in \Omega: w_{k}\left(x_{\alpha}, x_{3}\right) \neq u_{n_{k}}\left(x_{\alpha}, \varepsilon_{n_{k}} x_{3}\right)\right\}\right) \\
& =\mathcal{L}^{3}\left(\left\{x \in \Omega: z_{k}\left(\left(F_{i_{0}}^{\left(n_{k}\right)}\right)^{-1}\left(x_{\alpha}, \varepsilon_{n_{k}} x_{3}\right)\right) \neq \bar{u}_{n_{k}}\left(\left(F_{i_{0}}^{\left(n_{k}\right)}\right)^{-1}\left(x_{\alpha}, \varepsilon_{n_{k}} x_{3}\right)\right)\right\}\right) \\
& =\frac{1}{\varepsilon_{n_{k}}} \mathcal{L}^{3}\left(\left\{y \in S_{0}^{\left(n_{k}\right)}: z_{k}\left(\left(F_{i_{0}}^{\left(n_{k}\right)}\right)^{-1}(y)\right) \neq \bar{u}_{n_{k}}\left(\left(F_{i_{0}}^{\left(n_{k}\right)}\right)^{-1}(y)\right)\right\}\right) \\
& =\frac{1}{\varepsilon_{n_{k}}} \mathcal{L}^{3}\left(\left\{y \in S_{0}^{\left(n_{k}\right)}:\left(F_{i_{0}}^{\left(n_{k}\right)}\right)^{-1}(y) \in E_{k}\right\}\right)=\frac{1}{\varepsilon_{n_{k}}} \mathcal{L}^{3}\left(\left(F_{i_{0}}^{\left(n_{k}\right)}\right)^{-1}\left(S_{0}^{\left(n_{k}\right)}\right) \cap E_{k}\right) \\
& =\frac{1}{\varepsilon_{n_{k}}} \mathcal{L}^{3}\left(S_{i_{0}}^{\left(n_{k}\right)} \cap E_{k}\right) \leq 2 \mathcal{L}^{3}\left(E_{k}\right) \rightarrow 0 \text { as } k \rightarrow \infty,
\end{aligned}
$$

where we have used the fact that $i_{0} \in I_{k} \subset I_{\star}$.

In order to prove (ii), i.e. the equi-integrability of $\left\{\left|\left(\nabla_{\alpha} w_{k} \mid \frac{1}{\varepsilon_{n_{k}}} \nabla_{3} w_{k}\right)\right|^{p}\right\}$, let $A$ be any Borel subset of $\Omega$, and set

$$
A_{k}:=\left\{y \in S_{0}^{\left(n_{k}\right)}: y_{\alpha}=x_{\alpha}, y_{3}=\varepsilon_{n_{k}} x_{3}, x \in A\right\}
$$


and

$$
\bar{A}_{k}:=\left(F_{i_{0}}^{\left(n_{k}\right)}\right)^{-1}\left(A_{k}\right)
$$

Changing variables, by (3.3) and (3.7) we have

$$
\begin{aligned}
\int_{A}\left|\left(\nabla_{\alpha} w_{k} \mid \frac{1}{\varepsilon_{n_{k}}} \nabla_{3} w_{k}\right)\left(x_{\alpha}, x_{3}\right)\right|^{p} \mathrm{~d} x & =\frac{1}{\varepsilon_{n_{k}}} \int_{\bar{A}_{k}}\left|\nabla z_{k}(x)\right|^{p} \mathrm{~d} x \leq 8 \sum_{j \in I_{k}} \int_{\bar{A}_{k}}\left|\nabla z_{k}(x)\right|^{p} \mathrm{~d} x \\
& =8 \sum_{j \in I_{k}} \int_{\left(G_{j}^{\left(i_{0}, k\right)}\right)^{-1}\left(\bar{A}_{k}\right)}\left|\nabla z_{k}\left(G_{j}^{\left(i_{0}, k\right)}(y)\right)\right|^{p} \mathrm{~d} y .
\end{aligned}
$$

Splitting the last integral into a sum of two integrals over $\left(G_{j}^{\left(i_{0}, k\right)}\right)^{-1}\left(\bar{A}_{k}\right) \cap\left(\Omega \backslash\left(X^{\left(i_{0}, k\right)} \cap S_{j}^{\left(n_{k}\right)}\right)\right)$ and $\left(G_{j}^{\left(i_{0}, k\right)}\right)^{-1}\left(\bar{A}_{k}\right) \cap\left(X^{\left(i_{0}, k\right)} \cap S_{j}^{\left(n_{k}\right)}\right)$ respectively, using the definition of $X^{\left(i_{0}, k\right)}$ and the fact that

$$
\mathcal{L}^{3}\left(\bigcup_{j \in I_{k}}\left(G_{j}^{\left(i_{0}, k\right)}\right)^{-1}\left(\bar{A}_{k}\right)\right) \leq\left(\operatorname{card} I_{k}\right) \varepsilon_{n_{k}} \mathcal{L}^{3}(A) \leq \mathcal{L}^{3}(A)
$$

we have

$$
\begin{aligned}
& \sum_{j \in I_{k}} \int_{\left(G_{j}^{\left(i_{0}, k\right)}\right)^{-1}\left(\bar{A}_{k}\right) \cap\left(\Omega \backslash\left(X^{\left(i_{0}, k\right)} \cap S_{j}^{\left(n_{k}\right)}\right)\right)}\left|\nabla z_{k}\left(G_{j}^{\left(i_{0}, k\right)}(y)\right)\right|^{p} \mathrm{~d} y \leq \bigcup_{j \in I_{k}}\left(G_{j}^{\left(i_{0}, k\right)}\right)^{-1}\left(\bar{A}_{k}\right) \\
& \leq \Lambda\left(\mathcal{L}^{3}(A)\right),
\end{aligned}
$$

and in view of (3.8) we obtain

$$
\int_{A}\left|\left(\nabla_{\alpha} w_{k} \mid \frac{1}{\varepsilon_{n_{k}}} \nabla_{3} w_{k}\right)\left(x_{\alpha}, x_{3}\right)\right|^{p} \mathrm{~d} x \leq 8\left(\Lambda\left(\mathcal{L}^{3}(A)\right)+a_{k}\right) .
$$

The equi-integrability of $\left\{\left|\left(\nabla_{\alpha} w_{k} \mid \frac{1}{\varepsilon_{n_{k}}} \nabla_{3} w_{k}\right)\right|^{p}\right\}$ now follows from (3.5).

Step 4 (Proof of Claim 1). The selection of $i_{0} \in I_{k}$ will rest on De Giorgi's slicing argument to identify a layer of small energy concentration via an averaging process. We start by proving that for each $k \in \mathbb{N}$ and $i, j \in I_{k}$,

$$
X^{(i, k)} \cap S_{j}^{\left(n_{k}\right)} \subset\left(E_{k} \cap S_{j}^{\left(n_{k}\right)}\right) \cup\left(\left(G_{j}^{(i, k)}\right)^{-1}\left(E_{k} \cap S_{i}^{\left(n_{k}\right)}\right)\right) .
$$

Indeed, if $x \in\left(X^{(i, k)} \cap S_{j}^{\left(n_{k}\right)}\right) \backslash\left(E_{k} \cap S_{j}^{\left(n_{k}\right)}\right)$, then

$$
\bar{u}_{n_{k}}(x)=z_{k}(x) \neq z_{k}\left(G_{j}^{(i, k)}(x)\right),
$$

and

$$
\bar{u}_{n_{k}}(x)=\bar{u}_{n_{k}}\left(G_{j}^{(i, k)}(x)\right),
$$


where we have used the vertical periodicity of $\bar{u}_{n_{k}}$ and the fact that $i, j \in I_{k}$ are even. Thus

$$
\bar{u}_{n_{k}}\left(G_{j}^{(i, k)}(x)\right) \neq z_{k}\left(G_{j}^{(i, k)}(x)\right) \text {, i.e. } G_{j}^{(i, k)}(x) \in E_{k} \cap S_{i}^{\left(n_{k}\right)},
$$

and we conclude that

$$
\left(X^{(i, k)} \cap S_{j}^{\left(n_{k}\right)}\right) \backslash\left(E_{k} \cap S_{j}^{\left(n_{k}\right)}\right) \subset\left(G_{j}^{(i, k)}\right)^{-1}\left(E_{k} \cap S_{i}^{\left(n_{k}\right)}\right),
$$

thus proving (3.12). By the definition of $I_{k}$, and in view of (3.3), we have

$$
\mathcal{L}^{3}\left(\bigcup_{i \in I_{k}} G_{j}^{(i, k)}\left(E_{k} \cap S_{j}^{\left(n_{k}\right)}\right)\right) \leq \sum_{i \in I_{k}} \mathcal{L}^{3}\left(E_{k} \cap S_{j}^{\left(n_{k}\right)}\right) \leq 2 \mathcal{L}^{3}\left(E_{k}\right) \rightarrow 0 \text { as } k \rightarrow+\infty,
$$

and

$$
\mathcal{L}^{3}\left(\bigcup_{i \in I_{k}}\left(E_{k} \cap S_{i}^{\left(n_{k}\right)}\right)\right) \leq 2 \mathcal{L}^{3}\left(E_{k}\right) \rightarrow 0 \text { as } k \rightarrow+\infty
$$

We obtain

$$
\begin{aligned}
& \sum_{i \in I_{k}} \sum_{j \in I_{k}} \int_{G_{j}^{(i, k)}}\left|\nabla z_{k}(x)\right|^{p} \mathrm{~d} x \leq \sum_{i \in I_{k}} \sum_{j \in I_{k}} \int_{\left.G_{j}^{(i, k)} \cap S_{j}^{\left(n_{k}\right)}\right)} \int_{\left(E_{k} \cap S_{j}^{\left(n_{k}\right)}\right) \cup\left(E_{k} \cap S_{i}^{\left(n_{k}\right)}\right)}\left|\nabla z_{k}(x)\right|^{p} \mathrm{~d} x \\
& \leq \sum_{j \in I_{k}}\left(\sum_{i \in I_{k}} \int_{G_{j}^{(i, k)}}\left|\nabla z_{k}(x)\right|^{p} \mathrm{~d} x\right)+\sum_{\left.j \in I_{k} \cap S_{j}^{\left(n_{k}\right)}\right)} \sum_{i \in I_{k}} \int_{E_{k} \cap S_{i}^{\left(n_{k}\right)}}\left|\nabla z_{k}(x)\right|^{p} \mathrm{~d} x \\
& \leq \sum_{j \in I_{k}} \int_{i \in I_{k}} \int_{G_{j}^{(i, k)}\left(E_{k} \cap S_{j}^{\left(n_{k}\right)}\right)}\left|\nabla z_{k}(x)\right|^{p} \mathrm{~d} x+\frac{1}{\varepsilon_{n_{k}}} \int_{i \in I_{k}} \int_{\left(E_{k} \cap S_{i}^{\left(n_{k}\right)}\right)}\left|\nabla z_{k}(x)\right|^{p} \mathrm{~d} x \\
& \leq \frac{2}{\varepsilon_{n_{k}}} \Lambda\left(2 \mathcal{L}^{3}\left(E_{k}\right)\right) \text {. }
\end{aligned}
$$

Define $a_{k}:=16 \Lambda\left(2 \mathcal{L}^{3}\left(E_{k}\right)\right)$. In view of (3.13), we deduce the existence of an index $i_{0} \in I_{k}$ that satisfies (3.8). Note that by (3.5) and since $\mathcal{L}^{3}\left(E_{k}\right) \rightarrow 0$ as $k \rightarrow+\infty$, we have that $a_{k} \rightarrow 0$ as $k \rightarrow+\infty$, thus proving our Claim 1.

Step 5: The remaining of the proof is dedicated to establishing (iii) and (iv). By (ii) it follows that

$$
\left\{\nabla w_{k}\right\} \text { is bounded in } L^{p}\left(\Omega, \mathbb{R}^{9}\right),
$$

so in order to ensure the weak convergence of (a subsequence of) $\left\{w_{k}\right\}$ in $W^{1, p}\left(\Omega, \mathbb{R}^{3}\right)$ we first need to prove that

$$
\sup _{k \in \mathbb{N}} \int_{\Omega}\left|w_{k}(x)\right|^{p} \mathrm{~d} x<+\infty
$$


Since $\mathcal{L}^{3}\left(M_{k}\right) \rightarrow 0$ as $k \rightarrow \infty$ (see $\left.(3.11)\right)$, there exists $k_{1} \in \mathbb{N}$ such that

$$
\mathcal{L}^{3}\left(\left\{x \in \Omega:\left(w_{k}-v_{n_{k}}\right)(x)=0\right\}\right) \geq \frac{\mathcal{L}^{3}(\Omega)}{2},
$$

for all $k \geq k_{1}$. By virtue of Lemma 2.2 there exists a constant $C(\Omega)>0$ such that

$$
\int_{\Omega}\left|w_{k}(x)-v_{n_{k}}(x)\right|^{p} \mathrm{~d} x \leq C(\Omega) \int_{\Omega}\left|\nabla w_{k}(x)-\nabla v_{n_{k}}(x)\right|^{p} \mathrm{~d} x \text { for all } k \geq k_{1},
$$

and thus, using (3.14) and the fact that $\left\{v_{n}\right\}$ is bounded in $W^{1, p}\left(\Omega, \mathbb{R}^{3}\right)$ we obtain (3.15).

Since the sequence $\left\{w_{k}\right\}$ is bounded in $W^{1, p}\left(\Omega ; \mathbb{R}^{3}\right)$, up to a subsequence (not relabelled) it converges weakly in $W^{1, p}\left(\Omega ; \mathbb{R}^{3}\right)$ to some function $w \in W^{1, p}\left(\Omega ; \mathbb{R}^{3}\right)$, and (i) entails (iii), i.e. $w=v \mathcal{L}^{3}$ a.e. $x \in \Omega$ (note also that the full sequence $\left\{w_{k}\right\}$ must converge weakly to $\left.v\right)$.

It remains to prove (iv). In what follows we will denote by $[a]_{\beta}, \beta \in\{1,2,3\}$ the components of $a \in \mathbb{R}^{3}$. Let $\varphi \in L^{p \prime}(\Omega)$ be given.

We have

$$
\begin{aligned}
\int_{\Omega}\left[\frac{1}{\varepsilon_{n_{k}}} \nabla_{3} w_{k}(x)\right]_{\beta} \varphi(x) \mathrm{d} x= & \int_{\Omega \backslash M_{k}}\left[\frac{1}{\varepsilon_{n_{k}}} \nabla_{3} v_{n_{k}}(x)\right]_{\beta} \varphi(x) \mathrm{d} x \\
& +\int_{M_{k}}\left[\frac{1}{\varepsilon_{n_{k}}} \nabla_{3} w_{k}(x)\right]_{\beta} \varphi(x) \mathrm{d} x \\
= & \int_{\Omega}\left[\frac{1}{\varepsilon_{n_{k}}} \nabla_{3} v_{n_{k}}(x)\right]_{\beta} \varphi(x) \mathrm{d} x \\
& +\int_{M_{k}}\left[\frac{1}{\varepsilon_{n_{k}}} \nabla_{3} w_{k}(x)-\frac{1}{\varepsilon_{n_{k}}} \nabla_{3} v_{n_{k}}(x)\right]_{\beta} \varphi(x) \mathrm{d} x .
\end{aligned}
$$

Since $\frac{1}{\varepsilon_{n}} \nabla_{3} v_{n} \rightarrow b$ weakly in $L^{p}\left(\Omega, \mathbb{R}^{3}\right)$, we have

$$
\int_{\Omega}\left[\frac{1}{\varepsilon_{n_{k}}} \nabla_{3} v_{n_{k}}(x)\right]_{\beta} \varphi(x) \mathrm{d} x \rightarrow \int_{\Omega}[b(x)]_{\beta} \varphi(x) \mathrm{d} x \text { as } j \rightarrow \infty .
$$

On the other hand, by (ii) the sequence $\left\{\left|\left[\frac{1}{\varepsilon_{n_{k}}} \nabla_{3} w_{k}\right]_{\beta}\right|^{p}\right\}$ is equi-integrable, hence bounded in $L^{1}(\Omega)$, and by Hölder's inequality we obtain

$$
\begin{aligned}
& \left.\left|\int_{M_{k}}\left[\frac{1}{\varepsilon_{n_{k}}} \nabla_{3} w_{k}(x)-\frac{1}{\varepsilon_{n_{k}}} \nabla_{3} v_{n_{k}}(x)\right]_{\beta} \varphi(x) \mathrm{d} x\right|^{1 / p}\left(\int_{M_{k}}\left|\left[\frac{1}{\varepsilon_{n_{k}}} \nabla_{3} w_{k}(x)-\frac{1}{\varepsilon_{n_{k}}} \nabla_{3} v_{n_{k}}(x)\right]_{\beta}\right|^{p}(x) \mathrm{d} x\right)^{1 / p^{\prime}}|\varphi(x)|^{p \prime} \mathrm{d} x\right)^{M_{k}} \\
& \leq\left\|\left[\frac{1}{\varepsilon_{n_{k}}} \nabla_{3} w_{k}(x)-\frac{1}{\varepsilon_{n_{k}}} \nabla_{3} v_{n_{k}}(x)\right]_{\beta}\right\|_{L^{p}(\Omega)}\left(\int_{M_{k}}|\varphi(x)|^{p \prime} \mathrm{d} x\right)^{1 / p^{\prime}} \rightarrow 0 \text { as } j \rightarrow \infty,
\end{aligned}
$$


where we have used (3.11). We conclude that

$$
\int_{\Omega}\left[\frac{1}{\varepsilon_{n_{k}}} \nabla_{3} w_{k}(x)\right]_{\beta} \varphi(x) \mathrm{d} x \rightarrow \int_{\Omega}[b(x)]_{\beta} \varphi(x) \mathrm{d} x \text { as } j \rightarrow \infty
$$

for every $\varphi \in L^{p \prime}(\Omega)$ and each $\beta \in\{1,2,3\}$, and the proof of (iv) is complete.

It is possible to prove the relative weak compactness of the sequence $\left\{w_{k}\right\}$ in $W^{1, p}\left(\Omega ; \mathbb{R}^{3}\right)$ without making use of Lemma 2.2 (see Step 5 above). We will use a different approach, following closely the construction of the sequence $\left\{z_{k}\right\}$ in Step 1. To this end, we recall a proof of Proposition 2.1 hinged on the two propositions below.

Proposition 3.1. ([16] Cor. 3.3.3) Let $\Omega \in \mathbb{R}^{N}$ be an open and bounded set and let $\left\{u_{n}\right\}$ be a sequence of functions uniformly bounded in $L^{1}(\Omega)$. For $\lambda>0$ consider the truncation $\tau_{\lambda}: \mathbb{R} \rightarrow \mathbb{R}$ defined by

$$
\tau_{\lambda}(z):= \begin{cases}z & \text { if }|z| \leq \lambda, \\ \frac{z}{|z|} \lambda & \text { if }|z|>\lambda .\end{cases}
$$

Then there exists a subsequence of $\left\{u_{n}\right\}$ (not relabelled) and an increasing sequence of numbers $\lambda_{n} \rightarrow+\infty$ such that the truncated sequence $\left\{\tau_{\lambda_{n}} \circ u_{n}\right\}$ is equi-integrable and

$$
\lim _{n \rightarrow \infty} \mathcal{L}^{N}\left(\left\{x \in \Omega: u_{n}(x) \neq\left(\tau_{\lambda_{n}} \circ u_{n}\right)(x)\right\}\right)=0 .
$$

Proposition 3.2. (see $[1,2,26])$ Let $p>1$ and let $w \in W^{1, p}\left(\mathbb{R}^{N}, \mathbb{R}^{m}\right)$. Given $\lambda>0$ there exists a Lipschitz function $z$ in $\mathbb{R}^{N}$ such that $w=z \mathcal{L}^{N}$ a.e. on $\left\{x \in \mathbb{R}^{N}: M(\nabla w)(x)<\lambda\right\}$ and the Lipschitz constant of $z$ is bounded by $C(N) \lambda$, where $C(N)$ is a constant depending only upon dimension.

We now turn to the construction of the sequence $\left\{z_{k}\right\}$. As $\Omega$ is a bounded, Lipschitz domain we can extend the functions $\bar{u}_{n}$ (see Step 1 ) to $\mathbb{R}^{3} \backslash \Omega$ in such a way that the extensions $U_{n} \in W^{1, p}\left(\mathbb{R}^{3}, \mathbb{R}^{3}\right)$ satisfy

$$
\left\|U_{n}\right\|_{W^{1, p}\left(\mathbb{R}^{3}, \mathbb{R}^{3}\right)} \leq C\left\|\bar{u}_{n}\right\|_{W^{1, p}\left(\Omega, \mathbb{R}^{3}\right)},
$$

for some $C>0$. Therefore, by (3.4) and (2.1) we have

$$
\sup _{n \in \mathbb{N}} \int_{\mathbb{R}^{3}}\left|M\left(\nabla U_{n}\right)\right|^{p} \mathrm{~d} x<+\infty .
$$

Since the sequence $\left\{\left|M\left(\nabla U_{n}\right)\right|^{p}\right\}$ is uniformly bounded in $L^{1}(\Omega)$, Proposition 3.1 now guarantees the existence of a subsequence $\left\{\left|M\left(\nabla U_{n_{k}}\right)\right|^{p}\right\}$ of $\left\{\left|M\left(\nabla U_{n}\right)\right|^{p}\right\}$ and of an increasing sequence $\left\{\lambda_{k}\right\}$, with $\lambda_{k} \nearrow+\infty$, such that the truncated sequence $\left\{\tau_{\lambda_{k}} \circ\left|M\left(\nabla U_{n_{k}}\right)\right|^{p}\right\}$ is equi-integrable and

$$
\lim _{k \rightarrow \infty} \mathcal{L}^{3}\left(\left\{x \in \Omega:\left|M\left(\nabla U_{n_{k}}\right)\right|^{p}(x) \neq\left(\tau_{\lambda_{k}} \circ\left|M\left(\nabla U_{n_{k}}\right)\right|^{p}\right)(x)\right\}\right)=0 .
$$

By Proposition 3.2, for each $k \in \mathbb{N}$ there exists $z_{k} \in W^{1, \infty}\left(\mathbb{R}^{3} ; \mathbb{R}^{3}\right)$ such that $z_{k}=U_{n_{k}} \mathcal{L}^{3}$ a.e. on $\mathbb{R}^{3} \backslash R_{k}$ and $\operatorname{Lip}\left(z_{k}\right) \leq C \lambda_{k}^{\frac{1}{p}}$ (and thus $\left|\nabla z_{k}(x)\right| \leq C \lambda_{k}^{\frac{1}{p}}$ for $\mathcal{L}^{3}$ a.e. $x \in \mathbb{R}^{3}$ ), where

$$
R_{k}:=\left\{x \in \mathbb{R}^{3}: M\left(\nabla U_{n_{k}}\right)(x) \geq \lambda_{k}^{\frac{1}{p}}\right\}
$$


and $C>0$ is a constant. Note that

$$
E_{k}=\left\{x \in \Omega: z_{k}(x) \neq U_{n_{k}}(x)\right\}
$$

where the set $E_{k}$ has been defined in Step 1. The definition of $R_{k}$ and (3.16) imply that

$$
\mathcal{L}^{3}\left(E_{k}\right) \leq \mathcal{L}^{3}\left(R_{k} \cap \Omega\right) \leq \frac{1}{\lambda_{k}} \int_{\Omega}\left|M\left(\nabla U_{n_{k}}\right)(x)\right|^{p} \mathrm{~d} x \rightarrow 0 \text { as } k \rightarrow \infty .
$$

To prove that the sequence $\left\{\left|\nabla z_{k}\right|^{p}\right\}$ is equi-integrable, note that for $\mathcal{L}^{3}$ a.e. $x \in \Omega \backslash R_{k}$ we have

$$
\begin{aligned}
\left|\nabla z_{k}(x)\right|^{p} & =\left|\nabla U_{n_{k}}(x)\right|^{p} \leq\left|M\left(\nabla U_{n_{k}}\right)(x)\right|^{p} \\
& =\left(\tau_{\lambda_{k}} \circ\left|M\left(\nabla U_{n_{k}}\right)\right|^{p}\right)(x),
\end{aligned}
$$

while for $\mathcal{L}^{3}$ a.e. $x \in \Omega \cap R_{k}$,

$$
\left|\nabla z_{k}(x)\right|^{p} \leq C^{p} \lambda_{k}=C^{p}\left(\tau_{\lambda_{k}} \circ\left|M\left(\nabla U_{n_{k}}\right)\right|^{p}\right)(x) .
$$

Thus,

$$
\left|\nabla z_{k}(x)\right|^{p} \leq \max \left\{1, C^{p}\right\}\left(\tau_{\lambda_{k}} \circ\left|M\left(\nabla U_{n_{k}}\right)\right|^{p}\right)(x) \text { for } \mathcal{L}^{3} \text { a.e. } x \in \Omega
$$

and the equi-integrability of $\left\{\left|\nabla z_{k}\right|^{p}\right\}$ follows from the equi-integrability of $\left\{\tau_{\lambda_{k}} \circ\left|M\left(\nabla U_{n_{k}}\right)\right|^{p}\right\}$. For each $k \in \mathbb{N}$, define the Lipschitz function $w_{k}$ and the set $M_{k}$ as in Step 3.

To assert the relative weak compactness of $\left\{w_{k}\right\}$ in $W^{1, p}\left(\Omega ; \mathbb{R}^{3}\right)$ we first need to prove that

$$
\sup _{j \in \mathbb{N}} \int_{\Omega}\left|w_{k_{j}}(x)\right|^{p} \mathrm{~d} x<+\infty
$$

for some subsequence $\left\{w_{k_{j}}\right\}$ of $\left\{w_{k}\right\}$. To this end, we claim that there exists a subsequence $\left\{M_{k_{j}}\right\}$ of $\left\{M_{k}\right\}$ such that

$$
\mathcal{L}^{3}\left(\bigcap_{j \in \mathbb{N}}\left(\Omega \backslash M_{k_{j}}\right)\right)>0
$$

where the sets $M_{k}$ have been introduced in (3.10).

Since $\mathcal{L}^{3}\left(M_{k}\right) \rightarrow 0$ as $k \rightarrow \infty($ see $(3.11)$ and $(3.17))$, there exists $k_{1} \in \mathbb{N}$ such that $\mathcal{L}^{3}\left(\Omega \backslash M_{k}\right)>\frac{\mathcal{L}^{3}(\Omega)}{2}$ for all $k \geq k_{1}$, and thus

$$
\mathcal{L}^{3}\left(\Omega \backslash M_{k_{1}}\right)-\mathcal{L}^{3}\left(M_{k_{1}}\right)>0
$$

Let $k_{2}>k_{1}$ be such that $\mathcal{L}^{3}\left(M_{k_{2}}\right)<\frac{\mathcal{L}^{3}\left(M_{k_{1}}\right)}{2}$. Since $\Omega \backslash M_{k_{1}} \subset M_{k_{2}} \cup\left(\left(\Omega \backslash M_{k_{1}}\right) \cap\left(\Omega \backslash M_{k_{2}}\right)\right)$, we have

$$
\mathcal{L}^{3}\left(\left(\Omega \backslash M_{k_{1}}\right) \cap\left(\Omega \backslash M_{k_{2}}\right)\right) \geq \mathcal{L}^{3}\left(\Omega \backslash M_{k_{1}}\right)-\frac{\mathcal{L}^{3}\left(M_{k_{1}}\right)}{2} .
$$

Inductively, we construct a sequence $k_{j} \rightarrow \infty$ such that

$$
\mathcal{L}^{3}\left(\bigcap_{i=1}^{j}\left(\Omega \backslash M_{k_{i}}\right)\right) \geq \mathcal{L}^{3}\left(\Omega \backslash M_{k_{1}}\right)-\mathcal{L}^{3}\left(M_{k_{1}}\right) \sum_{i=1}^{j} \frac{1}{2^{i}}
$$


and letting $j \rightarrow \infty$ we obtain

$$
\mathcal{L}^{3}\left(\bigcap_{i=1}^{\infty}\left(\Omega \backslash M_{k_{i}}\right)\right) \geq \mathcal{L}^{3}\left(\Omega \backslash M_{k_{1}}\right)-\mathcal{L}^{3}\left(M_{k_{1}}\right),
$$

which, together with (3.20), establishes (3.19).

Since $v_{n_{k_{j}}} \rightarrow v$ weakly in $W^{1, p}\left(\Omega ; \mathbb{R}^{3}\right)$, there exists a subsequence (not relabelled) such that $v_{n_{k_{j}}}(x) \rightarrow v(x) \mathcal{L}^{3}$ a.e. $x \in \Omega$. Set $N:=\left\{x \in \Omega: v_{n_{k_{j}}}(x)\right.$ does not converge to $\left.v(x)\right\}$. By virtue of (3.19) and since $\mathcal{L}^{3}(N)=0$ we deduce that

$$
\left(\bigcap_{j=1}^{\infty}\left(\Omega \backslash M_{k_{j}}\right)\right) \cap(\Omega \backslash N) \neq \emptyset
$$

and we choose an element of this intersection, say $x_{0}$. Note that

$$
w_{k_{j}}\left(x_{0}\right)=v_{n_{k_{j}}}\left(x_{0}\right) \rightarrow v\left(x_{0}\right) \text { as } j \rightarrow \infty .
$$

Since $w_{k_{j}}$ is a Lipschitz function and $\operatorname{Lip}\left(w_{k_{j}}\right) \leq C \lambda_{k_{j}}^{1 / p}$ (see (3.9)), we have

$$
\left|w_{k_{j}}(x)\right| \leq\left|w_{k_{j}}\left(x_{0}\right)\right|+C \lambda_{k_{j}}^{1 / p}\left|x-x_{0}\right| \text { for all } x \in \Omega,
$$

and thus

$$
\begin{aligned}
\int_{M_{k_{j}}}\left|w_{k_{j}}(x)\right|^{p} \mathrm{~d} x & \leq 2^{p-1}\left|w_{k_{j}}\left(x_{0}\right)\right|^{p} \mathcal{L}^{3}\left(M_{k_{j}}\right)+2^{p-1} C^{p} \lambda_{k_{j}} \int_{M_{k_{j}}}\left|x-x_{0}\right|^{p} \mathrm{~d} x \\
& \leq 2^{p-1}\left|w_{k_{j}}\left(x_{0}\right)\right|^{p} \mathcal{L}^{3}\left(M_{k_{j}}\right)+C \lambda_{k_{j}} \mathcal{L}^{3}\left(M_{k_{j}}\right) \\
& \leq 2^{p}\left|w_{k_{j}}\left(x_{0}\right)\right|^{p} \mathcal{L}^{3}\left(E_{k_{j}}\right)+C \int_{\Omega}\left|M\left(\nabla U_{n_{k_{j}}}\right)(x)\right|^{p} \mathrm{~d} x
\end{aligned}
$$

where we have used (3.11) and (3.17). By the definition of $M_{k_{j}}$, we have

$$
\int_{\Omega}\left|w_{k_{j}}(x)\right|^{p} \mathrm{~d} x=\int_{\Omega \backslash M_{k_{j}}}\left|v_{n_{k_{j}}}(x)\right|^{p} \mathrm{~d} x+\int_{M_{k_{j}}}\left|w_{k_{j}}(x)\right|^{p} \mathrm{~d} x \leq\left\|v_{n_{k_{j}}}\right\|_{L^{p}\left(\Omega ; \mathbb{R}^{3}\right)}^{p}+\int_{M_{k_{j}}}\left|w_{k_{j}}(x)\right|^{p} \mathrm{~d} x,
$$

which, together with (3.16) and (3.21), asserts (3.18). Thus, taking into account (3.14), the sequence $\left\{w_{k_{j}}\right\}$ is bounded in $W^{1, p}\left(\Omega ; \mathbb{R}^{3}\right)$ and therefore $\left\{w_{k}\right\}$ is relatively weakly compact in $W^{1, p}\left(\Omega ; \mathbb{R}^{3}\right)$.

\section{Proof of Corollary 1.2}

To show that $Q_{\omega}^{*} W\left(u_{0}\right)=Q \bar{W}\left(\xi_{0}\right)$, let $(u, L)$ be admissible for $Q_{\omega}^{*} W\left(u_{0}\right)$. Using a density argument and in view of (1.1), without loss of generality we may assume that $u \in W^{1, p}\left(\Omega ; \mathbb{R}^{3}\right) \cap C^{\infty}\left(\bar{\Omega} ; \mathbb{R}^{3}\right)$. The very definition of $\bar{W}$ (see (2.3)), equation (2.4), and Fubini's theorem yield

$$
\frac{1}{\mathcal{L}^{3}(\Omega)} \int_{\Omega} W\left(\nabla_{\alpha} u \mid L \nabla_{3} u\right) \mathrm{d} x \geq Q \bar{W}\left(\xi_{0}\right)
$$


and thus,

$$
Q_{\omega}^{*} W\left(u_{0}\right) \geq Q \bar{W}\left(\xi_{0}\right)
$$

Conversely, let us fix $\delta>0$. In view of (2.5), there exist $\varepsilon_{n}^{\delta} \rightarrow 0^{+}$and $\left\{u_{n}^{\delta}\right\} \subset W^{1, p}\left(\Omega ; \mathbb{R}^{3}\right)$, with $u_{n \mid \partial \omega \times(-1,1)}^{\delta}=u_{0}$ and $u_{n}^{\delta} \rightarrow u_{0}$ strongly in $L^{p}\left(\Omega ; \mathbb{R}^{3}\right)$, such that

$$
\begin{aligned}
Q \bar{W}\left(\xi_{0}\right)+\delta & \geq \liminf _{n \rightarrow \infty} \frac{1}{\mathcal{L}^{3}(\Omega)} \int_{\Omega} W\left(\nabla_{\alpha} u_{n}^{\delta} \mid \frac{1}{\varepsilon_{n}^{\delta}} \nabla_{3} u_{n}^{\delta}\right) \mathrm{d} x \\
& \geq Q_{\omega}^{*} W\left(u_{0}\right),
\end{aligned}
$$

and letting $\delta \rightarrow 0^{+}$we obtain

$$
Q \bar{W}\left(\xi_{0}\right) \geq Q_{\omega}^{*} W\left(u_{0}\right)
$$

This, together with (4.1), asserts (1.3).

Step 1: We will first treat the particular case where $\omega=Q_{c}^{\prime}:=(-c / 2, c / 2)^{2}$, with $c>0$ fixed. Let $\left\{\left(v_{n}, L_{n}\right)\right\} \subset$ $W^{1, p}\left(\Omega ; \mathbb{R}^{3}\right) \times(0,+\infty)$ be an infimizing sequence for $Q_{\omega}^{*} W\left(u_{0}\right)$, and extend $v_{n}-u_{0}$ by periodicity to $\mathbb{R}^{2} \times(-1,1)$. Now let $\left\{\varepsilon_{n}\right\}$ be any sequence of positive numbers converging to zero. Define $v_{n, k}: \Omega \rightarrow \mathbb{R}^{3}$ by

$$
v_{n, k}(x):=\varepsilon_{k} L_{n}\left(v_{n}-u_{0}\right)\left(\left(\varepsilon_{k} L_{n}\right)^{-1} x_{\alpha}, x_{3}\right) .
$$

By the Riemann-Lebesgue lemma, we have that

$$
\lim _{n \rightarrow \infty} \lim _{k \rightarrow \infty}\left\|v_{n, k}\right\|_{L^{p}\left(\Omega ; \mathbb{R}^{3}\right)}=0,
$$

and

$$
\lim _{n \rightarrow \infty} \lim _{k \rightarrow \infty} \frac{1}{\mathcal{L}^{3}(\Omega)} \int_{\Omega} W\left(\nabla_{\alpha} u_{0}+\nabla_{\alpha} v_{n, k} \mid \frac{1}{\varepsilon_{k}} \nabla_{3} v_{n, k}\right) \mathrm{d} x=Q_{\omega}^{*} W\left(u_{0}\right) .
$$

Using a diagonalization argument, we can construct an increasing sequence $\{k(n)\} \subseteq \mathbb{N}$, with $k(n) \nearrow+\infty$ as $n \rightarrow+\infty$, such that

$$
\lim _{n \rightarrow \infty}\left\|\widetilde{v}_{n}\right\|_{L^{p}\left(\Omega ; \mathbb{R}^{3}\right)}=0
$$

and

$$
\lim _{n \rightarrow \infty} \frac{1}{\mathcal{L}^{3}(\Omega)} \int_{\Omega} W\left(\nabla_{\alpha} u_{0}+\nabla_{\alpha} \widetilde{v}_{n} \mid \frac{1}{\widetilde{\varepsilon}_{n}} \nabla_{3} \widetilde{v}_{n}\right) \mathrm{d} x=Q_{\omega}^{*} W\left(u_{0}\right),
$$

where $\widetilde{v}_{n}:=v_{n, k(n)}$ and $\widetilde{\varepsilon}_{n}:=\varepsilon_{k(n)}$. Thus, in view of (1.1),

$$
\sup _{n \in \mathbb{N}}\left\|\widetilde{v}_{n}\right\|_{W^{1, p}\left(\Omega, \mathbb{R}^{3}\right)}<+\infty
$$

and up to a subsequence (not relabelled), by (4.2) we have that $\widetilde{v}_{n} \rightarrow 0$ weakly in $W^{1, p}\left(\Omega ; \mathbb{R}^{3}\right)$. By Theorem 1.1 we deduce that there exist a subsequence $\left\{\widetilde{v}_{n_{k}}\right\}$ of $\left\{\widetilde{v}_{n}\right\}$ and a sequence $\left\{w_{k}\right\} \subset W^{1, \infty}\left(\Omega ; \mathbb{R}^{3}\right)$ such that

$$
\lim _{k \rightarrow \infty} \mathcal{L}^{3}\left(\widetilde{M}_{k}\right)=0,
$$


with $\widetilde{M}_{k}:=\left\{x \in \Omega: \widetilde{v}_{n_{k}}(x) \neq w_{k}(x)\right\}$,

$$
\lim _{k \rightarrow \infty}\left\|w_{k}\right\|_{L^{p}\left(\Omega ; \mathbb{R}^{3}\right)}=0
$$

and

$$
\left\{\left(\nabla_{\alpha} w_{k} \mid \frac{1}{\widetilde{\varepsilon}_{n_{k}}} \nabla_{3} w_{k}\right)\right\} \text { is p-equi-integrable. }
$$

By (1.1) we have that

$$
\begin{aligned}
\int_{\Omega} W\left(\nabla_{\alpha} u_{0}+\nabla_{\alpha} w_{k} \mid \frac{1}{\widetilde{\varepsilon}_{n_{k}}} \nabla_{3} w_{k}\right) \mathrm{d} x \leq & \int_{\Omega} W\left(\nabla_{\alpha} u_{0}+\nabla_{\alpha} \widetilde{v}_{n_{k}} \mid \frac{1}{\widetilde{\varepsilon}_{n_{k}}} \nabla_{3} \widetilde{v}_{n_{k}}\right) \mathrm{d} x \\
& +\int_{\widetilde{M}_{k}} W\left(\nabla_{\alpha} u_{0}+\nabla_{\alpha} w_{k} \mid \frac{1}{\widetilde{\varepsilon}_{n_{k}}} \nabla_{3} w_{k}\right) \mathrm{d} x+C \mathcal{L}^{3}\left(\widetilde{M}_{k}\right),
\end{aligned}
$$

and thus, in view of $(4.3,4.4)$ and $(4.6)$,

$$
\limsup _{k \rightarrow \infty} \frac{1}{\mathcal{L}^{3}(\Omega)} \int_{\Omega} W\left(\nabla_{\alpha} u_{0}+\nabla_{\alpha} w_{k} \mid \frac{1}{\widetilde{\varepsilon}_{n_{k}}} \nabla_{3} w_{k}\right) \mathrm{d} x \leq Q_{\omega}^{*} W\left(u_{0}\right) .
$$

For each $j \in \mathbb{N}$ define $\omega_{j}:=\left\{x \in \omega:\right.$ dist $\left.(x, \partial \omega)<\frac{1}{j}\right\}$, and consider cut-off functions $\theta_{j} \in C_{c}^{\infty}(\omega ;[0,1])$ such that $\theta_{j}$ vanishes in a neighborhood of $\partial \omega, \theta_{j} \equiv 1$ in $\omega \backslash \omega_{j}$, and $\left\|\nabla \theta_{j}\right\|_{L^{\infty}\left(\omega ; \mathbb{R}^{2}\right)} \leq C j$, for some constant $C>0$. Define $w_{k, j}: \Omega \rightarrow \mathbb{R}^{3}$ by

$$
w_{k, j}\left(x_{\alpha}, x_{3}\right):=u_{0}\left(x_{\alpha}\right)+\theta_{j}\left(x_{\alpha}\right) w_{k}\left(x_{\alpha}, x_{3}\right) .
$$

Since by (4.5) we have that

$$
\lim _{j \rightarrow \infty} \lim _{k \rightarrow \infty}\left\|w_{k, j}-u_{0}\right\|_{L^{p}\left(\Omega ; \mathbb{R}^{3}\right)}=0
$$

a diagonalization argument provides an increasing sequence $k(j) \nearrow+\infty$ as $j \rightarrow+\infty$ such that

$$
\lim _{j \rightarrow \infty}\left\|u_{j}-u_{0}\right\|_{L^{p}\left(\Omega ; \mathbb{R}^{3}\right)}=0
$$

and

$$
\left\|w_{k(j)}\right\|_{L^{p}\left(\Omega ; \mathbb{R}^{3}\right)}^{\frac{1}{2}} \leq \frac{1}{j}
$$

where we have denoted $w_{k(j), j}$ by $u_{j}$. Set $\bar{\varepsilon}_{j}:=\widetilde{\varepsilon}_{n_{k(j)}}$. Note that we have

$$
\nabla_{\alpha} u_{j}\left(x_{\alpha}, x_{3}\right)=\xi_{0}+\nabla \theta_{j}\left(x_{\alpha}\right) \otimes w_{k(j)}\left(x_{\alpha}, x_{3}\right)+\theta_{j}\left(x_{\alpha}\right) \nabla_{\alpha} w_{k(j)}\left(x_{\alpha}, x_{3}\right),
$$

and

$$
\nabla_{3} u_{j}\left(x_{\alpha}, x_{3}\right)=\theta_{j}\left(x_{\alpha}\right) \nabla_{3} w_{k(j)}\left(x_{\alpha}, x_{3}\right)
$$


Thus,

$$
\int_{A}\left|\left(\nabla_{\alpha} u_{j} \mid \frac{1}{\bar{\varepsilon}_{j}} \nabla_{3} u_{j}\right)\right|^{p} \mathrm{~d} x \leq C\left(\left|\xi_{0}\right|^{p} \mathcal{L}^{3}(A)+j^{p} \int_{A}\left|w_{k(j)}\right|^{p} \mathrm{~d} x+\int_{A}\left|\left(\nabla_{\alpha} w_{k(j)} \mid \frac{1}{\bar{\varepsilon}_{j}} \nabla_{3} w_{k(j)}\right)\right|^{p} \mathrm{~d} x\right),
$$

for all Borel subsets $A$ of $\Omega$, and by (4.6) and (4.8) we deduce that (iv) holds (after relabelling). It remains to prove that (i) is satisfied. To this end, let us define

$$
T_{j}:=\left\{x \in \Omega: u_{j}(x) \neq w_{k(j)}(x)+u_{0}\left(x_{\alpha}\right)\right\},
$$

and note that

$$
\mathcal{L}^{3}\left(T_{j}\right) \leq \mathcal{L}^{3}\left(\omega_{j}\right) \rightarrow 0 \text { as } j \rightarrow \infty
$$

We have,

$$
\begin{aligned}
\int_{\Omega} W\left(\nabla_{\alpha} u_{j} \mid \frac{1}{\bar{\varepsilon}_{j}} \nabla_{3} u_{j}\right) \mathrm{d} x= & \int_{\Omega} W\left(\nabla_{\alpha} u_{0}+\nabla_{\alpha} w_{k(j)} \mid \frac{1}{\bar{\varepsilon}_{j}} \nabla_{3} w_{k(j)}\right) \mathrm{d} x+\int_{T_{j}} W\left(\nabla_{\alpha} u_{j} \mid \frac{1}{\bar{\varepsilon}_{j}} \nabla_{3} u_{j}\right) \mathrm{d} x \\
& -\int_{T_{j}} W\left(\nabla_{\alpha} u_{0}+\nabla_{\alpha} w_{k(j)} \mid \frac{1}{\bar{\varepsilon}_{j}} \nabla_{3} w_{k(j)}\right) \mathrm{d} x
\end{aligned}
$$

and since the last two integrands are equi-integrable (use (1.1)), equations (4.7) and (4.9) imply that

$$
\limsup _{j \rightarrow \infty} \frac{1}{\mathcal{L}^{3}(\Omega)} \int_{\Omega} W\left(\nabla_{\alpha} u_{j} \mid \frac{1}{\bar{\varepsilon}_{j}} \nabla_{3} u_{j}\right) \mathrm{d} x \leq Q_{\omega}^{*} W\left(u_{0}\right)
$$

As $\left(u_{j}, \frac{1}{\bar{\varepsilon}_{j}}\right)$ is admissible for $Q_{\omega}^{*} W\left(u_{0}\right)$, we conclude that

$$
\lim _{j \rightarrow \infty} \frac{1}{\mathcal{L}^{3}(\Omega)} \int_{\Omega} W\left(\nabla_{\alpha} u_{j} \mid \frac{1}{\bar{\varepsilon}_{j}} \nabla_{3} u_{j}\right) \mathrm{d} x=Q_{\omega}^{*} W\left(u_{0}\right)
$$

thus asserting (i).

Step 2: In this part of the proof we remove the restriction of $\omega$ being a cube. Set $Q^{\prime}:=(-1 / 2,1 / 2)^{2}$, and let

$$
\omega^{(m)}:=\bigcup_{i=1}^{N(m)}\left(a_{i}+L_{i} Q^{\prime}\right) \subset \subset \omega
$$

be a union of pairwise disjoint cubes such the $\mathcal{L}^{2}\left(\omega \backslash \omega^{(m)}\right) \leq \frac{1}{m}$, where $m \in \mathbb{N}$, and $L_{i}>0(i=1, \cdots, N(m))$. Since the definition of $Q_{\omega}^{*} W\left(u_{0}\right)$ is invariant under translations of the projection $\omega$ of the domain of integration, given a sequence $\left\{\varepsilon_{n}\right\}$ of positive real numbers converging to zero we can repeatedly apply Step 1 to each cube $a_{i}+L_{i} Q^{\prime}$, thus finding a subsequence $\left\{\varepsilon_{n, m}\right\}$ of $\left\{\varepsilon_{n}\right\}$ and sequences $\left\{u_{n}^{(i)}\right\} \subset W^{1, p}\left(\left(a_{i}+L_{i} Q^{\prime}\right) \times(-1,1) ; \mathbb{R}^{3}\right)(i=$ $1, \cdots, N(m)$ ), such that (i-iii) hold (with $\omega, u_{n}$ and $\varepsilon_{n}$ replaced by $a_{i}+L_{i} Q^{\prime}, u_{n}^{(i)}$ and $\varepsilon_{n, m}$, respectively). Define $u_{n, m} \in W^{1, p}\left(\Omega ; \mathbb{R}^{3}\right)$ by

$$
u_{n, m}\left(x_{\alpha}, x_{3}\right):=\sum_{i=1}^{N(m)} u_{n}^{(i)}\left(x_{\alpha}, x_{3}\right) \chi_{\left(a_{i}+L_{i} Q^{\prime}\right) \times(-1,1)}\left(x_{\alpha}, x_{3}\right)+u_{0}\left(x_{\alpha}\right) \chi_{\left(\omega \backslash \omega^{(m)}\right) \times(-1,1)}\left(x_{\alpha}, x_{3}\right) .
$$


Note that $u_{n, m \mid \partial \omega \times(-1,1)}=u_{0}$, and that we have

$$
\lim _{m \rightarrow \infty} \lim _{n \rightarrow \infty}\left\|u_{n, m}-u_{0}\right\|_{L^{p}\left(\Omega ; \mathbb{R}^{3}\right)}^{p}=\lim _{m \rightarrow \infty} \lim _{n \rightarrow \infty} \sum_{i=1}^{N(m)}\left\|u_{n}^{(i)}-u_{0}\right\|_{L^{p}\left(\left(a_{i}+L_{i} Q^{\prime}\right) \times(-1,1) ; \mathbb{R}^{3}\right)}^{p}=0 .
$$

In view of (1.1) we obtain

$$
\begin{aligned}
& \frac{1}{\mathcal{L}^{3}(\Omega)} \int_{\Omega} W\left(\nabla_{\alpha} u_{n, m} \mid \frac{1}{\varepsilon_{n, m}} \nabla_{3} u_{n, m}\right) \mathrm{d} x \\
& =\frac{1}{\mathcal{L}^{3}(\Omega)}\left(\sum_{i=1}^{N(m)} \int_{\left(a_{i}+L_{i} Q^{\prime}\right) \times(-1,1)} W\left(\nabla_{\alpha} u_{n}^{(i)} \mid \frac{1}{\varepsilon_{n, m}} \nabla_{3} u_{n}^{(i)}\right) \mathrm{d} x+\int_{(\omega \backslash \omega(m)) \times(-1,1)} W\left(\xi_{0} \mid 0\right) \mathrm{d} x\right) \\
& \leq \frac{1}{\mathcal{L}^{3}(\Omega)}\left(\sum_{i=1}^{N(m)} \int_{\left(a_{i}+L_{i}\right.} W\left(\nabla_{\alpha} u_{n}^{(i)} \mid \frac{1}{\varepsilon_{n, m}} \nabla_{3} u_{n}^{(i)}\right) \mathrm{d} x+\frac{2 C\left(1+\left|\xi_{0}\right|^{p}\right)}{m}\right),
\end{aligned}
$$

and thus,

$$
\limsup _{m \rightarrow \infty} \limsup _{n \rightarrow \infty} \frac{1}{\mathcal{L}^{3}(\Omega)} \int_{\Omega} W\left(\nabla_{\alpha} u_{n, m} \mid \frac{1}{\varepsilon_{n, m}} \nabla_{3} u_{n, m}\right) \mathrm{d} x \leq Q \bar{W}\left(\xi_{0}\right) .
$$

By the usual diagonalization argument we may find $n(m) \nearrow+\infty$ as $m \rightarrow+\infty$, such that

$$
\lim _{m \rightarrow \infty}\left\|u_{n(m), m}-u_{0}\right\|_{L^{p}\left(\Omega ; \mathbb{R}^{3}\right)}=0
$$

and

$$
\limsup _{m \rightarrow \infty} \frac{1}{\mathcal{L}^{3}(\Omega)} \int_{\Omega} W\left(\nabla_{\alpha} u_{n(m), m} \mid \frac{1}{\varepsilon_{n(m), m}} \nabla_{3} u_{n(m), m}\right) \mathrm{d} x \leq Q \bar{W}\left(\xi_{0}\right) .
$$

Since (1.3) holds and as $\left(u_{n(m), m}, \frac{1}{\varepsilon_{n(m), m}}\right)$ is admissible for $Q_{\omega}^{*} W\left(u_{0}\right)$, we obtain that

$$
\lim _{m \rightarrow \infty} \frac{1}{\mathcal{L}^{3}(\Omega)} \int_{\Omega} W\left(\nabla_{\alpha} u_{n(m), m} \mid \frac{1}{\varepsilon_{n(m), m}} \nabla_{3} u_{n(m), m}\right) \mathrm{d} x=Q \bar{W}\left(\xi_{0}\right) .
$$

It remains to prove the $p$-equi-integrability required in (iv). While at this stage this is not necessarily satisfied, we can nevertheless mimic the arguments used in Step 1 (first apply Th. 1.1 to get a $p$-equi-integrable sequence, then modify the new sequence near the lateral boundary $\partial \omega \times(-1,1))$ to construct a sequence $\left\{u_{n}\right\} \subset W^{1, p}\left(\Omega ; \mathbb{R}^{3}\right)$ and a subsequence of $\left\{\varepsilon_{n}\right\}$ such that (i-iv) hold simultaneously. The details of this construction are identical to those of the construction performed in Step 1, and we will not repeat them here.

\section{Proof of Corollary 1.3}

The proof is strongly motivated by that of Proposition 6.3 (Step 1) in [13]. Theorem 1.1 implies that there exist a subsequence $\left\{v_{n(k)}\right\}$ of $\left\{v_{n}\right\}$ and a sequence $\left\{\widetilde{w}_{k}\right\} \subset W^{1, \infty}\left(\Omega ; \mathbb{R}^{3}\right)$ such that $\lim _{n \rightarrow \infty} \mathcal{L}^{3}\left(\left\{x \in \Omega: \widetilde{w}_{k}(x) \neq\right.\right.$ 
$\left.\left.v_{n(k)}(x)\right\}\right)=0, \lim _{k \rightarrow \infty}\left\|\widetilde{w}_{k}-u_{0}\right\|_{L^{p}\left(\Omega ; \mathbb{R}^{3}\right)}=0$ and the sequence $\left\{\left(\nabla_{\alpha} \widetilde{w}_{k} \mid \frac{1}{\varepsilon_{n(k)}} \nabla_{3} \widetilde{w}_{k}\right)\right\}$ is $p$-equi-integrable. Thus, taking into account (1.1) and using the fact that $\left\{\left(v_{n(k)}, \varepsilon_{n(k)}\right)\right\}$ is a recovering sequence, we obtain that

$$
\limsup _{k \rightarrow+\infty} \int_{\Omega} W\left(\nabla_{\alpha} \widetilde{w}_{k} \mid \frac{1}{\varepsilon_{n(k)}} \nabla_{3} \widetilde{w}_{k}\right) \mathrm{d} x \leq 2 \int_{\omega} Q \bar{W}\left(\nabla_{\alpha} u_{0}\left(x_{\alpha}\right)\right) \mathrm{d} x_{\alpha} .
$$

Repeating the arguments used in the proof of Corollary 1.2, we may modify $\widetilde{w}_{k}$ appropriately near the lateral boundary $\partial \omega \times(-1,1)$ so that we obtain subsequences $\left\{\bar{\varepsilon}_{j}\right\}$ and $\left\{\bar{v}_{j}\right\}$ of $\left\{\varepsilon_{n(k)}\right\}$ and $\left\{v_{n(k)}\right\}$ respectively, and a sequence $\left\{u_{j}\right\} \subset W^{1, p}\left(\Omega ; \mathbb{R}^{3}\right)$ such that

$$
\begin{gathered}
\lim _{j \rightarrow \infty} \mathcal{L}^{3}\left(\left\{x \in \Omega: u_{j}(x) \neq \bar{v}_{j}(x)\right\}\right)=0, \\
\lim _{j \rightarrow \infty}\left\|u_{j}-u_{0}\right\|_{L^{p}\left(\Omega ; \mathbb{R}^{3}\right)}=0 \\
\left\{\left(\nabla_{\alpha} u_{j} \mid \frac{1}{\bar{\varepsilon}_{j}} \nabla_{3} u_{j}\right)\right\} \quad \text { is } p \text {-equi-integrable }
\end{gathered}
$$

and

$$
u_{j \mid \partial \omega \times(-1,1)}=u_{0}
$$

hold. Moreover, in view of $(2.5,5.1)$ and $(5.4)$, we have that

$$
\lim _{j \rightarrow \infty} \int_{\Omega} W\left(\nabla_{\alpha} u_{j} \mid \frac{1}{\bar{\varepsilon}_{j}} \nabla_{3} u_{j}\right) \mathrm{d} x=2 \int_{\omega} Q \bar{W}\left(\nabla_{\alpha} u_{0}\left(x_{\alpha}\right)\right) \mathrm{d} x_{\alpha}
$$

Let us define $\Omega_{k}^{+}:=\omega \times\left(1-\frac{1}{k}, 1\right), \Omega_{k}^{-}:=\omega \times\left(-1,-1+\frac{1}{k}\right)$, and put $\Omega_{k}:=\Omega_{k}^{+} \cup \Omega_{k}^{-}$. Changing variables, we obtain that

$$
\int_{\Omega \backslash \Omega_{k}} W\left(\nabla_{\alpha} u_{j} \mid \frac{1}{\bar{\varepsilon}_{j}} \nabla_{3} u_{j}\right) \mathrm{d} x=\left(1-\frac{1}{k}\right) \int_{\Omega} W\left(\nabla_{\alpha} v_{j, k} \mid \frac{1}{\bar{\varepsilon}_{j}\left(1-\frac{1}{k}\right)} \nabla_{3} v_{j, k}\right) \mathrm{d} x
$$

where $v_{j, k}: \Omega \rightarrow \mathbb{R}^{3}$ is defined by $v_{j, k}\left(x_{\alpha}, x_{3}\right):=u_{j}\left(x_{\alpha},\left(1-\frac{1}{k}\right) x_{3}\right)$. Note that by (5.5) we have $v_{j, k \mid \partial \omega \times(-1,1)}$ $=u_{0}$, and also

$$
\begin{aligned}
\left\|v_{j, k}-u_{0}\right\|_{L^{p}\left(\Omega ; \mathbb{R}^{3}\right)}^{p} & =\left(1-\frac{1}{k}\right)^{-1} \int_{\Omega \backslash \Omega_{k}}\left|u_{j}\left(y_{\alpha}, y_{3}\right)-u_{0}\left(y_{\alpha}\right)\right|^{p} \mathrm{~d} y_{\alpha} \mathrm{d} y_{3} \\
& \leq\left(1-\frac{1}{k}\right)^{-1}\left\|u_{j}-u_{0}\right\|_{L^{p}\left(\Omega ; \mathbb{R}^{3}\right)}^{p} .
\end{aligned}
$$

Thus,

$$
\lim _{j \rightarrow \infty}\left\|v_{j, k}-u_{0}\right\|_{L^{p}\left(\Omega ; \mathbb{R}^{3}\right)}=0 \text { for all } k \geq 2,
$$


and we deduce that for each integer $k \geq 2$ fixed the sequence $\left\{\left(v_{j, k},\left(1-\frac{1}{k}\right) \bar{\varepsilon}_{j}\right)\right\}$ is admissible for the infimum in (2.5). This implies that we have

$$
\liminf _{j \rightarrow \infty} \int_{\Omega} W\left(\nabla_{\alpha} v_{j, k} \mid \frac{1}{\left(1-\frac{1}{k}\right) \bar{\varepsilon}_{j}} \nabla_{3} v_{j, k}\right) \mathrm{d} x \geq 2 \int_{\omega} Q \bar{W}\left(\nabla_{\alpha} u_{0}\left(x_{\alpha}\right)\right) \mathrm{d} x_{\alpha},
$$

and in view of (5.6) and (5.7), we obtain that

$$
\begin{aligned}
& \limsup _{j \rightarrow \infty} \int_{\Omega_{k}} W\left(\nabla_{\alpha} u_{j} \mid \frac{1}{\bar{\varepsilon}_{j}} \nabla_{3} u_{j}\right) \mathrm{d} x \\
& =\limsup _{j \rightarrow \infty}\left(\int_{\Omega} W\left(\nabla_{\alpha} u_{j} \mid \frac{1}{\bar{\varepsilon}_{j}} \nabla_{3} u_{j}\right) \mathrm{d} x-\left(1-\frac{1}{k}\right) \int_{\Omega} W\left(\nabla_{\alpha} v_{j, k} \mid \frac{1}{\left(1-\frac{1}{k}\right) \bar{\varepsilon}_{j}} \nabla_{3} v_{j, k}\right) \mathrm{d} x\right) \\
& \leq 2 \int_{\omega} Q \bar{W}\left(\nabla_{\alpha} u_{0}\left(x_{\alpha}\right)\right) \mathrm{d} x_{\alpha}-\liminf _{j \rightarrow \infty}\left(1-\frac{1}{k}\right) \int_{\Omega} W\left(\nabla_{\alpha} v_{j, k} \mid \frac{1}{\left(1-\frac{1}{k}\right) \bar{\varepsilon}_{j}} \nabla_{3} v_{j, k}\right) \mathrm{d} x \\
& \leq \frac{2}{k} \int_{\omega} Q \bar{W}\left(\nabla_{\alpha} u_{0}\left(x_{\alpha}\right)\right) \mathrm{d} x_{\alpha} .
\end{aligned}
$$

Taking into account (1.1), we deduce that

$$
\limsup _{j \rightarrow \infty} \int_{\Omega_{k}}\left|\left(\nabla_{\alpha} u_{j} \mid \frac{1}{\bar{\varepsilon}_{j}} \nabla_{3} u_{j}\right)\right|^{p} \mathrm{~d} x \leq \frac{C}{k} \text { for all } k \geq 2,
$$

for some constant $C>0$.

Partition $\Omega_{k}^{-}$and $\Omega_{k}^{+}$into $k$ horizontal layers of height $\frac{1}{k^{2}}$, denoted by $L_{k, i}^{-}$and $L_{k, i}^{+}$respectively (indexed in a symmetric way with respect to the plane $\left.x_{3}=0 ; i=1, \cdots, k\right)$. In view of $(5.8)$, for each integer $k \geq 2$ there exists $J(k) \in \mathbb{N}$ such that

$$
\sum_{i=1}^{k} \int_{L_{k, i}^{-} \cup L_{k, i}^{+}}\left|\left(\nabla_{\alpha} u_{j} \mid \frac{1}{\bar{\varepsilon}_{j}} \nabla_{3} u_{j}\right)\right|^{p} \mathrm{~d} x \leq \frac{2 C}{k}, \text { for all } j \geq J(k) .
$$

Thus, for each $k \geq 2$ and $j \geq J(k)$, there exists an index $i(k, j) \in\{1, \cdots, k\}$ such that

$$
\int_{L_{k, i(k, j)}^{-} \cup L_{k, i(k, j)}^{+}}\left|\left(\nabla_{\alpha} u_{j} \mid \frac{1}{\bar{\varepsilon}_{j}} \nabla_{3} u_{j}\right)\right|^{p} \mathrm{~d} x \leq \frac{2 C}{k^{2}} .
$$

Put $L_{k, i(k, j)}^{-}:=\omega \times\left(-c_{k, j},-b_{k, j}\right)$ and $L_{k, i(k, j)}^{+}:=\omega \times\left(b_{k, j}, c_{k, j}\right)$. In particular, (5.9) yields

$$
\int_{-c_{k, j}}^{-\frac{b_{k, j}+c_{k, j}}{2}} \int_{\omega}\left|\left(\nabla_{\alpha} u_{j} \mid \frac{1}{\bar{\varepsilon}_{j}} \nabla_{3} u_{j}\right)\right|^{p} \mathrm{~d} x \leq \frac{2 C}{k^{2}}
$$


and

$$
\int_{\frac{b_{k, j}+c_{k, j}}{2}}^{c_{k, j}} \int_{\omega}\left|\left(\nabla_{\alpha} u_{j} \mid \frac{1}{\bar{\varepsilon}_{j}} \nabla_{3} u_{j}\right)\right|^{p} \mathrm{~d} x \leq \frac{2 C}{k^{2}}
$$

and thus, there exist $a_{k, j}^{-} \in\left(-c_{k, j},-\frac{b_{k, j}+c_{k, j}}{2}\right)$ and $a_{k, j}^{+} \in\left(\frac{b_{k, j}+c_{k, j}}{2}, c_{k, j}\right)$ such that we have

$$
\int_{\omega}\left|\left(\nabla_{\alpha} u_{j}\left(x_{\alpha}, a_{k, j}^{-}\right) \mid \frac{1}{\bar{\varepsilon}_{j}} \nabla_{3} u_{j}\left(x_{\alpha}, a_{k, j}^{-}\right)\right)\right|^{p} \mathrm{~d} x \leq 4 C,
$$

and

$$
\int_{\omega}\left|\left(\nabla_{\alpha} u_{j}\left(x_{\alpha}, a_{k, j}^{+}\right) \mid \frac{1}{\bar{\varepsilon}_{j}} \nabla_{3} u_{j}\left(x_{\alpha}, a_{k, j}^{+}\right)\right)\right|^{p} \mathrm{~d} x \leq 4 C .
$$

Consider smooth cut-off functions $\varphi_{k, j}=\varphi_{k, j}\left(x_{3}\right)$ such that

$$
\begin{gathered}
\varphi_{k, j} \equiv 1 \text { on }\left(a_{k, j}^{-}+\frac{1}{2 k^{2}}, a_{k, j}^{+}-\frac{1}{2 k^{2}}\right), \\
\varphi_{k, j} \equiv 0 \text { on }\left(-\infty,-\frac{b_{k, j}+c_{k, j}}{2}\right) \cup\left(\frac{b_{k, j}+c_{k, j}}{2},+\infty\right), \\
\left\|\varphi_{k, j}^{\prime}\right\|_{\infty} \leq c k^{2},
\end{gathered}
$$

and define $w_{k, j} \in W^{1, p}\left(\Omega ; \mathbb{R}^{3}\right)$ by

$$
\begin{aligned}
w_{k, j}\left(x_{\alpha}, x_{3}\right):= & \varphi_{k, j}\left(x_{3}\right) u_{j}\left(x_{\alpha}, x_{3}\right)+\left(1-\varphi_{k, j}\left(x_{3}\right)\right) u_{j}\left(x_{\alpha}, a_{k, j}^{-}\right) \chi_{\Omega_{k}^{-}}\left(x_{\alpha}, x_{3}\right) \\
& +\left(1-\varphi_{k, j}\left(x_{3}\right)\right) u_{j}\left(x_{\alpha}, a_{k, j}^{+}\right) \chi_{\Omega_{k}^{+}}\left(x_{\alpha}, x_{3}\right)
\end{aligned}
$$

It can be easily seen that

$$
w_{k, j \mid \partial \omega \times(-1,1)}=u_{0},
$$

and

$$
\lim _{k \rightarrow \infty} \limsup _{j \rightarrow \infty} \mathcal{L}^{3}\left(\left\{x \in \Omega: w_{k, j}(x) \neq u_{j}(x)\right\}\right)=0 .
$$

Claim: The following identities hold:

$$
\limsup _{j \rightarrow \infty}\left\|w_{k, j}-u_{0}\right\|_{L^{p}\left(\Omega ; \mathbb{R}^{3}\right)}=0 \text { for all } k \geq 2,
$$

and

$$
\lim _{k \rightarrow \infty} \limsup _{j \rightarrow \infty} \int_{\Omega_{k}}\left|\left(\nabla_{\alpha} w_{k, j} \mid \frac{1}{\bar{\varepsilon}_{j}} \nabla_{3} w_{k, j}\right)\right|^{p} \mathrm{~d} x=0 .
$$


Assuming that the claim holds, the usual diagonalization argument provides an increasing sequence $j(k) \nearrow \infty$ as $k \rightarrow \infty$, so that we have

$$
\begin{gathered}
\lim _{k \rightarrow \infty} \mathcal{L}^{3}\left(\left\{x \in \Omega: w_{k}(x) \neq u_{j(k)}(x)\right\}\right)=0 \\
\lim _{k \rightarrow \infty}\left\|w_{k}-u_{0}\right\|_{L^{p}\left(\Omega ; \mathbb{R}^{3}\right)}=0
\end{gathered}
$$

and

$$
\lim _{k \rightarrow \infty} \int_{\Omega_{k}}\left|\left(\nabla_{\alpha} w_{k} \mid \frac{1}{\varepsilon_{n_{k}}} \nabla_{3} w_{k}\right)\right|^{p} \mathrm{~d} x=0
$$

where we have denoted $w_{k}:=w_{k, j(k)}$ and $\varepsilon_{n_{k}}:=\bar{\varepsilon}_{j(k)}$. Note that the corresponding subsequence $\left\{v_{n_{k}}\right\}$ of $\left\{v_{n}\right\}$ is obtained by taking $v_{n_{k}}:=\bar{v}_{j(k)}$. Clearly (see (5.12)), we have that

$$
w_{k \mid \partial \omega \times(-1,1)}=u_{0} .
$$

In view of (5.2) and (5.16) we deduce that (i) holds, while (iii) follows from the particular way in which we have constructed the sequence $\left\{w_{k}\right\}$. To prove (ii), let $A$ be an arbitrary Borel subset of $\Omega$. We have

$$
\begin{aligned}
\int_{A}\left|\left(\nabla_{\alpha} w_{k} \mid \frac{1}{\varepsilon_{n_{k}}} \nabla_{3} w_{k}\right)\right|^{p} \mathrm{~d} x= & \int_{A \cap\left(\Omega \backslash \Omega_{k}\right)}\left|\left(\nabla_{\alpha} u_{j(k)} \mid \frac{1}{\bar{\varepsilon}_{j(k)}} \nabla_{3} u_{j(k)}\right)\right|^{p} \mathrm{~d} x \\
& +\int_{A \cap \Omega_{k}}\left|\left(\nabla_{\alpha} w_{k} \mid \frac{1}{\varepsilon_{n_{k}}} \nabla_{3} w_{k}\right)\right|^{p} \mathrm{~d} x \\
\leq & \int_{A}\left|\left(\nabla_{\alpha} u_{j(k)} \mid \frac{1}{\bar{\varepsilon}_{j(k)}} \nabla_{3} u_{j(k)}\right)\right|^{p} \mathrm{~d} x \\
& +\int_{\Omega_{k}}\left|\left(\nabla_{\alpha} w_{k} \mid \frac{1}{\varepsilon_{n_{k}}} \nabla_{3} w_{k}\right)\right|^{p} \mathrm{~d} x,
\end{aligned}
$$

and (ii) follows by (5.4) and (5.18).

We have

$$
\begin{aligned}
\int_{\Omega} W\left(\nabla_{\alpha} w_{k} \mid \frac{1}{\varepsilon_{n_{k}}} \nabla_{3} w_{k}\right) \mathrm{d} x= & \int_{\Omega} W\left(\nabla_{\alpha} u_{j(k)} \mid \frac{1}{\bar{\varepsilon}_{j(k)}} \nabla_{3} u_{j(k)}\right) \mathrm{d} x+\int_{\Omega_{k}} W\left(\nabla_{\alpha} w_{k} \mid \frac{1}{\varepsilon_{n_{k}}} \nabla_{3} w_{k}\right) \mathrm{d} x \\
& -\int_{\Omega_{k}} W\left(\nabla_{\alpha} u_{j(k)} \mid \frac{1}{\bar{\varepsilon}_{j(k)}} \nabla_{3} u_{j(k)}\right) \mathrm{d} x
\end{aligned}
$$

and thus, in view of (ii), equations $(1.1,5.4)$ and $(5.6)$, we obtain that

$$
\lim _{k \rightarrow \infty} \int_{\Omega} W\left(\nabla_{\alpha} w_{k} \mid \frac{1}{\varepsilon_{n_{k}}} \nabla_{3} w_{k}\right) \mathrm{d} x=2 \int_{\omega} Q \bar{W}\left(\nabla_{\alpha} u_{0}\left(x_{\alpha}\right)\right) \mathrm{d} x_{\alpha} .
$$

Taking now into account (5.17) and (5.19) we deduce that (iv) holds. 
Proof of Claim. We have

$$
\left\|w_{k, j}-u_{j}\right\|_{L^{p}\left(\Omega ; \mathbb{R}^{3}\right)}^{p} \leq \int_{\Omega_{k}^{-}}\left|u_{j}\left(x_{\alpha}, x_{3}\right)-u_{j}\left(x_{\alpha}, a_{k, j}^{-}\right)\right|^{p} \mathrm{~d} x+\int_{\Omega_{k}^{+}}\left|u_{j}\left(x_{\alpha}, x_{3}\right)-u_{j}\left(x_{\alpha}, a_{k, j}^{+}\right)\right|^{p} \mathrm{~d} x
$$

and by Hölder's inequality we obtain that

$$
\begin{aligned}
\int_{\Omega_{k}^{+}}\left|u_{j}\left(x_{\alpha}, x_{3}\right)-u_{j}\left(x_{\alpha}, a_{k, j}^{+}\right)\right|^{p} \mathrm{~d} x_{\alpha} \mathrm{d} x_{3} & =\iint_{\Omega_{k}^{+}}\left|\int_{x_{3}}^{a_{k, j}^{+}} \frac{\mathrm{d}}{\mathrm{d} s}\left(u_{j}\left(x_{\alpha}, s\right)\right) \mathrm{d} s\right|^{p} \mathrm{~d} x_{\alpha} \mathrm{d} x_{3} \\
& \leq \frac{1}{k^{\frac{p}{p^{\prime}}}} \int_{\Omega_{k}^{+}}\left(\int_{1-\frac{1}{k}}^{1}\left|\frac{\mathrm{d}}{\mathrm{d} s}\left(u_{j}\left(x_{\alpha}, s\right)\right)\right|^{p} \mathrm{~d} s\right) \mathrm{d} x \\
& =\frac{1}{k^{\frac{p}{p^{\prime}}+1}} \int_{\Omega_{k}^{+}}\left|\nabla_{3} u_{j}(x)\right|^{p} \mathrm{~d} x .
\end{aligned}
$$

Thus, in view of (5.8) we have that

$$
\begin{aligned}
\limsup _{j \rightarrow \infty} \int_{\Omega_{k}^{+}}\left|u_{j}\left(x_{\alpha}, x_{3}\right)-u_{j}\left(x_{\alpha}, a_{k, j}^{+}\right)\right|^{p} \mathrm{~d} x & \leq \frac{1}{k^{\frac{p}{p^{\prime}}+1}} \limsup _{j \rightarrow \infty}\left(\bar{\varepsilon}_{j}^{p} \int\left|\frac{1}{\Omega_{k}^{+}} \nabla_{3} u_{j}(x)\right|^{p} \mathrm{~d} x\right) \\
& \leq \frac{1}{k^{\frac{p}{p^{\prime}}+1}}\left(\limsup _{j \rightarrow \infty} \bar{\varepsilon}_{j}^{p}\right) \limsup _{j \rightarrow \infty} \int_{\Omega_{k}^{+}}\left|\frac{1}{\bar{\varepsilon}_{j}} \nabla_{3} u_{j}(x)\right|^{p} \mathrm{~d} x=0
\end{aligned}
$$

for each $k \geq 2$ fixed. Similarly, we obtain that

$$
\limsup _{j \rightarrow \infty} \int_{\Omega_{k}^{-}}\left|u_{j}\left(x_{\alpha}, x_{3}\right)-u_{j}\left(x_{\alpha}, a_{k, j}^{-}\right)\right|^{p} \mathrm{~d} x=0 \text { for all } k \geq 2 .
$$

Hence

$$
\limsup _{j \rightarrow \infty}\left\|w_{k, j}-u_{j}\right\|_{L^{p}\left(\Omega ; \mathbb{R}^{3}\right)}^{p}=0 \text { for all } k \geq 2
$$

which together with (5.3) yields (5.14). 
It remains to prove (5.15). To this end, we first note that we have

$$
\begin{aligned}
& \frac{1}{\bar{\varepsilon}_{j}^{p}} \int_{\Omega_{k}^{+}}\left|\varphi_{k, j}^{\prime}\left(x_{3}\right)\right|^{p}\left|u_{j}\left(x_{\alpha}, x_{3}\right)-u_{j}\left(x_{\alpha}, a_{k, j}^{+}\right)\right|^{p} \mathrm{~d} x_{\alpha} \mathrm{d} x_{3} \\
& \leq \frac{C k^{2 p}}{\bar{\varepsilon}_{j}^{p}} \int_{a_{k, j}^{+}-\frac{1}{2 k^{2}}}^{a_{k, j}^{+}} \int_{\omega}\left|u_{j}\left(x_{\alpha}, x_{3}\right)-u_{j}\left(x_{\alpha}, a_{k, j}^{+}\right)\right|^{p} \mathrm{~d} x_{\alpha} \mathrm{d} x_{3} \\
& \leq \frac{C k^{2 p}}{\bar{\varepsilon}_{j}^{p}} \int_{a_{k, j}^{+}-\frac{1}{2 k^{2}}}^{a_{k, j}^{+}}\left|\int_{x_{3}}^{a_{k, j}^{+}} \frac{\mathrm{d}}{\mathrm{d} s}\left(u_{j}\left(x_{\alpha}, x_{3}\right)\right) \mathrm{d} s\right|^{p} \mathrm{~d} x_{\alpha} \mathrm{d} x_{3} \\
& \leq \frac{C k^{2 p}}{k^{2 \frac{p}{p^{\prime}}} \bar{\varepsilon}_{j}^{p}} \int_{a_{k, j}^{+}-\frac{1}{2 k^{2}}}^{a_{k, j}^{+}} \int_{\omega}\left(\int_{a_{k, j}^{+}-\frac{1}{2 k^{2}}}^{a_{k, j}^{+}}\left|\frac{\mathrm{d}}{\mathrm{d} s} u_{j}\left(x_{\alpha}, s\right)\right|^{p} \mathrm{~d} s\right) \mathrm{d} x_{\alpha} \mathrm{d} x_{3} \\
& \leq \frac{C}{\bar{\varepsilon}_{j}^{p}} \int_{\Omega_{k}^{+}}\left|\nabla_{3} u_{j}(x)\right|^{p} \mathrm{~d} x,
\end{aligned}
$$

where we have used again Hölder's inequality. Thus, in view of (5.8) we deduce that

$$
\lim _{k \rightarrow \infty} \limsup _{j \rightarrow \infty} \frac{1}{\bar{\varepsilon}_{j}^{p}} \int_{\Omega_{k}^{+}}\left|\varphi_{k, j}^{\prime}\left(x_{3}\right)\right|^{p}\left|u_{j}\left(x_{\alpha}, x_{3}\right)-u_{j}\left(x_{\alpha}, a_{k, j}^{+}\right)\right|^{p} \mathrm{~d} x_{\alpha} \mathrm{d} x_{3}=0
$$

and also

$$
\lim _{k \rightarrow \infty} \limsup _{j \rightarrow \infty} \frac{1}{\bar{\varepsilon}_{j}^{p}} \int_{\Omega_{k}^{-}}\left|\varphi_{k, j}^{\prime}\left(x_{3}\right)\right|^{p}\left|u_{j}\left(x_{\alpha}, x_{3}\right)-u_{j}\left(x_{\alpha}, a_{k, j}^{-}\right)\right|^{p} \mathrm{~d} x_{\alpha} \mathrm{d} x_{3}=0
$$

We have

$$
\begin{aligned}
\int_{\Omega_{k}^{+}}\left|\left(\nabla_{\alpha} w_{k, j} \mid \frac{1}{\bar{\varepsilon}_{j}} \nabla_{3} w_{k, j}\right)\right|^{p} \mathrm{~d} x \leq & C \int_{\Omega_{k}^{+}}\left|\left(\nabla_{\alpha} u_{j} \mid \frac{1}{\bar{\varepsilon}_{j}} \nabla_{3} u_{j}\right)\right|^{p} \mathrm{~d} x+C \int_{\Omega_{k}^{+}}\left|\nabla_{\alpha} u_{j}\left(x_{\alpha}, a_{k, j}^{+}\right)\right|^{p} \mathrm{~d} x_{\alpha} \mathrm{d} x_{3} \\
& +\frac{C}{\bar{\varepsilon}_{j}^{p}} \int_{\Omega_{k}^{+}}\left|\varphi_{k, j}^{\prime}\left(x_{3}\right)\right|^{p}\left|u_{j}\left(x_{\alpha}, x_{3}\right)-u_{j}\left(x_{\alpha}, a_{k, j}^{+}\right)\right|^{p} \mathrm{~d} x_{\alpha} \mathrm{d} x_{3},
\end{aligned}
$$

and therefore, by virtue of $(5.8,5.11)$ and $(5.20)$ we obtain that

$$
\lim _{k \rightarrow \infty} \limsup _{j \rightarrow \infty} \int_{\Omega_{k}^{+}}\left|\left(\nabla_{\alpha} w_{k, j} \mid \frac{1}{\bar{\varepsilon}_{j}} \nabla_{3} w_{k, j}\right)\right|^{p} \mathrm{~d} x=0 .
$$


Similarly, invoking $(5.8,5.10)$ and $(5.21)$ we find

$$
\lim _{k \rightarrow \infty} \limsup _{j \rightarrow \infty} \int_{\Omega_{k}^{-}}\left|\left(\nabla_{\alpha} w_{k, j} \mid \frac{1}{\bar{\varepsilon}_{j}} \nabla_{3} w_{k, j}\right)\right|^{p} \mathrm{~d} x=0,
$$

thus asserting (5.15).

The authors thank Gilles Francfort for many stimulating discussions on the subject of this paper. The research of I. Fonseca was partially supported by the National Science Foundation under Grants Nos. DMS-9731957 and DMS0103798. The authors thank the Center for Nonlinear Analysis (NSF grant No. DMS-9803791), Carnegie Mellon University, Pittsburgh, PA, USA for its support during the preparation of this paper.

\section{REFERENCES}

[1] E. Acerbi and N. Fusco, Semicontinuity problems in the calculus of variations. Arch. Rational. Mech. Anal. 86 (1984) 125-145.

[2] E. Acerbi and N. Fusco, An approximation lemma for $W^{1, p}$ functions, in Material Instabilities in Continuum Mechanics and Related Mathematical Problems, edited by J.M. Ball. Heriot-Watt University, Oxford (1988).

[3] E. Anzelotti, S. Baldo and D. Percivale, Dimensional reduction in variational problems, asymptotic developments in $\Gamma$-convergence, and thin structures in elasticity. Asymptot. Anal. 9 (1994) 61-100.

[4] E.J. Balder, A general approach to lower semicontinuity and lower closure in optimal control theory. SIAM J. Control Optim. 22 (1984) 570-598.

[5] J.M. Ball, A version of the fundamental theorem for Young mesures, in PDE's and Continuum Models of Phase Transitions, edited by M. Rascle, D. Serre and M. Slemrod. Springer-Verlag, Berlin, Lecture Notes in Phys. 344 (1989) 207-215.

[6] H. Berliocchi and J.-M. Lasry, Intégrands normales et mesures paramétrées en calcul des variations. Bull. Soc. Math. France 101 (1973) 129-184.

[7] K. Bhattacharya and A. Braides, Thin films with many small cracks. Preprint (2000).

[8] K. Bhattacharya, I. Fonseca and G. Francfort, An asymptotic study of the debonding of thin films. Arch. Rational. Mech. Anal. 161 (2002) 205-229.

[9] K. Bhattacharya and R.D. James, A theory of thin films of martensitic materials with applications to microactuators. J. Mech. Phys. Solids 47 (1999) 531-576.

[10] A. Braides, Private communication.

[11] A. Braides, I. Fonseca and G. Francfort, 3D-2D asymptotic analysis for inhomogeneous thin films. Indiana Univ. Math. J. 49 (2000) 1367-1404.

[12] A. Braides and I. Fonseca, Brittle thin films. Appl. Math. Optim. 44 (2001) 299-323.

[13] S. Conti, I. Fonseca and G. Leoni, A $\Gamma$-convergence result for the two-gradient theory of phase transitions, Preprint 01-CNA008. Center for Nonlinear Analysis, Carnegie Mellon University (2001). Comm. Pure Applied Math. (to appear).

[14] B. Dacorogna, Direct Methods in the Calculus of Variations. Springer-Verlag (1989).

[15] I. Fonseca and G. Francfort, On the inadequacy of scaling of linear elasticity for 3D-2D asymptotics in a nonlinear setting. $J$. Math. Pures Appl. 80 (2001) 547-562.

[16] I. Fonseca and G. Leoni, Modern Methods in the Calculus of Variations with Applications to Nonlinear Continuum Physics. Springer-Verlag (to appear).

[17] I. Fonseca, S. Müller and P. Pedregal, Analysis of concentration and oscillation effects generated by gradients. SIAM J. Math. Anal. 29 (1998) 736-756.

[18] D.D. Fox, A. Raoult and J.C. Simo, A justification of nonlinear properly invariant plate theories. Arch. Rational. Mech. Anal. 124 (1993) 157-199.

[19] T. Iwaniec and C. Sbordone, On the integrability of the Jacobian under minimal hypotheses. Arch. Rational. Mech. Anal. 119 (1992) 129-143.

[20] D. Kinderlehrer and P. Pedregal, Characterizations of Young mesures generated by gradients. Arch. Rational. Mech. Anal. 115 (1991) 329-365.

[21] D. Kinderlehrer and P. Pedregal, Gradient Young mesures generated by sequences in Sobolev spaces. J. Geom. Anal. 4 (1994) 59-90.

[22] J. Kristensen, Finite functionals and Young measures generated by gradients of Sobolev functions. Mathematical Institute, Technical University of Denmark, Mat-Report No. 1994-34 (1994).

[23] J. Kristensen, Lower semicontinuity in spaces of weakly differentiable functions. Math. Ann. 313 (1999) 653-710.

[24] H. Le Dret and A. Raoult, The nonlinear membrane model as variational limit of nonlinear three-dimensional elasticity. $J$. Math. Pures Appl. 74 (1995) 549-578. 
[25] H. Le Dret and A. Raoult, Variational convergence for nonlinear shell models with directors and related semicontinuity and relaxation results. Arch. Rational. Mech. Anal. 154 (2000) 101-134.

[26] F.C. Liu, A Luzin type property of Sobolev functions. Indiana Univ. Math. J. 26 (1997) 645-651.

[27] P. Pedregal, Parametrized mesures and Variational Principles. Birkhäuser, Boston (1997).

[28] E.M. Stein, Singular integrals and differentiability properties of functions. Princeton University Press (1970).

[29] L. Tartar, Compensated compactness and applications to partial differential equations, in Nonlinear Analysis and Mechanics: Heriot-Watt Symposium, edited by R. Knops. Longman, Harlow, Pitman Res. Notes Math. Ser. 39 (1979) 136-212.

[30] L. Tartar, The compensated compactness method applied to systems of conservation laws, in Systems of Nonlinear Partial Differential Equations, edited by J.M. Ball. Riedel (1983).

[31] L. Tartar, Étude des oscillations dans les équations aux dérivées partielles nonlinéaires. Springer-Verlag, Berlin, Lecture Notes in Phys. 195 (1994) 384-412.

[32] Y.C. Shu, Heterogeneous thin films of martensitic materials. Arch. Rational. Mech. Anal. 153 (2000) 39-90.

[33] L.C. Young, Generalized curves and the existence of an attained absolute minimum in the calculus of variations. C. R. Soc. Sci. Lettres de Varsovie, Classe III 30 (1937) 212-234.

[34] L.C. Young, Lectures on the calculus of variations and optimal control theory. W.B. Saunders (1969).

[35] W.P. Ziemer, Weakly Differentiable Functions. Sobolev spaces and functions of bounded variation. Springer-Verlag, Berlin (1989). 